\title{
Post-Operative Fever Workup in Pediatric Neurosurgery Patients
}

\author{
Raviv, $\mathrm{N}^{1}$, Field, $\mathrm{N}^{1}$, Adamo, $\mathrm{MA}^{1}$ \\ ${ }^{1}$ Neurosurgery, Albany Medical Center, Albany, (New York) USA
}

\section{Introduction}

Fevers are common in the post-operative period, and literature indicates that workup for isolated fever is not warranted in the first two to four postoperative days. We sought to determine both the incidence of acute fever, and to assess the utility of performing further workup in the post-operative neurosurgical patients

\section{Methods}

A single-institution, retrospective study was performed examining patients age $</=18$ years with craniosynostosis, Chiari malformation, and brain tumors from 2009-2018. Fevers defined as a temperature $\geq 38.0^{\circ} \mathrm{C}$ were identified during the first four post-operative days. Charts were queried for urinalysis/culture (UA/Ucx), chest radiographs (CXR), blood cultures (BcX), cerebral spinal fluid culture (CSFCX), viral panel, white cell count (WBC), transfusion history, development of wound infection, and placement of ventricular(EVD) and lumbar drains(LD). 30day post-operative microbiology results and readmissions were reviewed. Descriptive statistics were performed using logistic regression analysis with IBM SPSS Statistics for Mac, version 25.

\section{Results}

235 patients were evaluated, and 61\% developed fevers within the first four post-operative days. $26.5 \%(38 / 143)$ of febrile patients underwent further workup, and patients with fevers $>39.0^{\circ} \mathrm{C}$ were more likely to undergo further evaluation, most commonly including UA/Ucx(21.7\%). $1 \%(2 / 143)$ of patients were found to have infection during the first 4 days, and 7 additional patients ( 6 with acute post-operative fevers) developed infection following the initial 4 days and within the first 30 post-operative days. linfectious complications were not found to correlate with acute post-operative fevers $(p<0.120)$; however, multiple days of acute post-operative fevers $(p<0.034)$ and the presence of EVDs $(p<0.001)$ were found to be associated with development of an infectious complication within 30-days. Acute post-operative fevers were associated with $\operatorname{EVD}(p<0.038)$, as well as blood product transfusions and an increased WBC count $(p<0.001)$.

\section{Conclusions}

Isolated fevers within the first four post-operative days are rarely associated with infectious etiology. Additional factors should be considered when deciding to pursue further investigation. 


\title{
Optical Coherence Tomography Can Detect Elevated Intracranial Pressure in Craniosynostosis
}

\author{
Shih-Shan Lang MD¹, Christopher Kalmar MD², Gregory Heuer MD, PhD ${ }^{1}$, Scott Bartlett MD², \\ Laura Humphries $\mathrm{MD}^{2}$, Phillip Storm $\mathrm{MD}^{1}$, Jesse Taylor $\mathrm{MD}^{2}$, Jordan Swanson $\mathrm{MD}^{2}$ \\ ${ }^{1}$ Division of Neurosurgery, Children's Hospital of Philadelphia, Department of Neurosurgery, University of \\ Pennsylvania, Perelman School of Medicine, Philadelphia, Pennsylvania \\ ${ }^{2}$ Division of Plastic \& Reconstructive Surgery, Children's Hospital of Philadelphia, Department of Surgery, \\ University of Pennsylvania, Perelman School of Medicine, Philadelphia, Pennsylvania
}

\section{Background}

Identifying elevated intracranial pressure (ICP) in patients with craniosynostosis may facilitate appropriate intervention in order to prevent neurocognitive impairments. Noninvasive spectraldomain optical coherence tomography (OCT) of the retina has demonstrated high sensitivity and specificity for detecting ICP. This study aimed to characterize patterns of elevated ICP among patients with craniosynostosis.

\section{Methods}

Quantitative retinal parameters using spectral-domain OCT were prospectively assessed bilaterally in patients with craniosynostosis. Subjects were considered to have elevated ICP if the invasive measurement was $\geq 15 \mathrm{mmHg}$. The threshold for retinal parameters equating to elevated ICP was retinal nerve fiber layer maximal thickness $>\mathbf{2 0 8}$ microns or maximal retinal inner projection $>159$ microns in either eye based on $95 \% \mathrm{Cl}$ of normal subjects. Univariate and multivariate analysis of OCT findings and type of craniosynostosis was performed.

\section{Results}

One hundred thirty-three subjects (age median 12.5 months, age range 1.0-205.3 months) with craniosynostosis were enrolled at the time of initial cranial vault expansion. Of these patients, $69.2 \%$ ( $n=92$ of 133 patients) had invasive ICP measurement intraoperatively and $80.4 \%$ ( $n=74$ of 92 patients) were nonsyndromic. There was a significant association between having elevated ICP based on suture involvement $(p=.047)$. Patients were more likely to have elevated ICP with unicoronal $(57.1 \%, n=4$ of 7$)$, sagittal $(51.4 \%, n=18$ of 35$)$, bicoronal $(100.0 \%, n=2$ of 2$)$, and multisuture involvement $(100 \%, n=5$ of 5$)$. Patients were more likely to have non-elevated ICP with metopic ( $68.2 \%, n=15$ of 22 ) and lambdoid $(66.7 \%, n=2$ of 3 ) synostosis. OCT demonstrated a sensitivity of $88 \%$ (95\% Cl 69\%-96\%) and specificity of $67 \%$ ( $95 \% \mathrm{Cl} 48-81 \%)$. 


\section{Conclusions}

OCT of the retina is a potentially sensitive and specific indicator of elevated ICP in craniosynostosis patients with normal fundoscopic exams and shows promise as a noninvasive diagnostic modality. Moreover, type of suture involved demonstrated significant association with predicting elevated ICP in nonsyndromic patients. 


\title{
Abstract \#4: Predictive Factors for Definitive CSF Diversion in
} Post-hemorrhagic Hydrocephalus

\author{
Ramin Eskandari ${ }^{1,2}$, Thomas Larrew ${ }^{1}$, Tyler Vasas ${ }^{1}$ \\ ${ }^{1}$ Department of Neurosurgery, Medical University of South Carolina, Charleston SC USA \\ ${ }^{2}$ Department of Pediatrics, Medical University of South Carolina, Charleston, SC USA
}

\section{Background}

Post-hemorrhagic hydrocephalus $(\mathrm{PHH})$ portends devastating outcomes in many preterm infants. Recent studies demonstrate a link between earlier intervention, smaller ventricles and haste for intraventricular hemorrhage removal to correlate with improved outcomes. We describe our use of early and aggressive reservoir intervention with a prolonged weaning period prior and how they correlate with CSF shunting.

\section{Methods}

34 Consecutive PHH infants with reservoirs between January 2016 and February, 2019 were evaluated. 26 patients met inclusion and underwent evaluation including multiple ventricular measurements, head circumference, weight, age and timing of reservoir placement among many other parameters to investigate the link between those weaned versus unweaned from reservoir tapping requiring CSF shunting. Standard tapping $=10 \mathrm{ml} / \mathrm{kg} / \mathrm{day}$. Shunting was offered at $\geq 2.5 \mathrm{~kg}$. Weaning was initiated at $\geq 38$ weeks gestation.

\section{Results}

Six patients were successfully weaned (follow-up 18-36 months). At last follow-up, 19 of 20 patients required ventriculoperitoneal shunting (VPS) (6-42 months). Birth weight (BW) and gestational age $(G A)$ were higher in weaned patients $(p=0.03)$. $3^{\text {rd }}$ ventricle $\left(3^{\text {rd }} V\right)$ and biventricular diameter (BVD) at $2.5 \mathrm{~kg}$ demonstrated weaning associations. Average BVD measured $31.4 \mathrm{~mm}$ and $40.6 \mathrm{~mm}$ in weaned versus unweaned respectively $(p=0.01)$. At $2.5 \mathrm{~kg}$, the $3^{\text {rd }} \mathrm{V}$ measured $6 \mathrm{~mm}$ and $8.2 \mathrm{~mm}$ in weaned versus unweaned $(p=0.03)$. Five of 6 weaned patients underwent prolonged tapping past shunting weight of $2.5 \mathrm{~kg}$.

\section{Conclusions}

Our small retrospective cohort demonstrates statistically significant differences in several factors in PHH newborns requiring shunting versus those successfully weaned. Although birth weight and age are important indicators of disease severity, ventricular size at the time of decision making for CSF shunting was significantly different in those who eventually were weaned. Multicenter cohorts with this aggressive and prolonged tapping/weaning protocol may establish predictive value of these metrics. These data also suggest a potential link between prolonged aggressive tapping and ultimate need for CSF diversion surgery.

\section{Author Disclosures}

Ramin Eskandari has received compensation for consulting work from Anuncia Inc. - unrelated to this research

Neither Thomas Larrew nor Tyler Vasas have any disclosures to report 


\title{
Short-term Functional Outcomes of Infra-Conus Mini-Laminotomy for Selective Dorsal Rhizotomy: A Surgical and Rehabilitative Perspective
}

\author{
Neena I. Marupudi ${ }^{1,3}$, M.D., M.S., Lubna Ayoubi, B.S. ${ }^{2,3}$, Melisa Concepcion, PA-C ${ }^{2,3}$, Charles \\ Pelshaw, M.D. ${ }^{2,3}$, Erika Erlandson, M.D. ${ }^{2,3}$ \\ ${ }^{1}$ Department of Pediatric Neurosurgery, Children's Hospital of Michigan, Detroit Medical Center \\ ${ }^{2}$ Department of Pediatric Physical Medicine and Rehabilitation, Children's Hospital of Michigan, \\ Detroit Medical Center \\ ${ }^{3}$ Wayne State University School of Medicine
}

\section{Background/Aims}

Selective dorsal rhizotomy (SDR) for the treatment of spasticity in children with cerebral palsy is an effective and well-validated surgical approach. While numerous techniques have been described in the past, including multiple level laminectomy and laminectomy at the conus, the infra-conus mini-laminectomy/laminotomy approach is another recently described approach. with advantages. Understanding the short-term effects on gross motor function and endurance postoperatively is necessary to prepare families and therapy staff regarding the expected progress in rehabilitation after surgery.

\section{Methods}

Patients undergoing SDR at the Children's Hospital of Michigan from 2017-2019 underwent preoperative and serial postoperative gross motor function, strength, and endurance testing. A linear mixed model with random intercept was used to estimate the period at which they regained their preoperative function levels. A linear spline model was utilized to investigate the rate of change in the short-term postoperative period while accounting for the expected functional decline in the initial post-operative period.

\section{Results}

Twenty-five patients who underwent SDR surgery and had at least 6 months of postoperative follow-up were investigated in this study. Gross motor function measure (GMFM) surpassed preoperative levels between 3 and 6 months after surgery. The rate of improvement in GMFM score was about 2.03 per month $(p<0.0001)$. The patients tended to surpass preoperative endurance measures (6-minute walk test) between 12 and 15 months after surgery. The rate of improvement in 6-minute walk test was about 62 steps per month from the 3-month follow-up visit up to 15 months ( $p=0.0006)$.

\section{Conclusions}

While there is a significant initial decline in endurance and gross motor function measures following surgery, this is followed by a significant increase in mobility and endurance that both reaches and surpasses preoperative levels. Patients tend to surpass their preoperative gross motor function status by 6 months and their endurance by 12 months following selective dorsal rhizotomy. 


\title{
Use of High-Density Subdural Grid to Increase Precision of Functional Mapping and Seizure Onset Zone Localization
}

\author{
Joffre Olaya, M.D., Maija-Riikka Steenari, M.D., William Loudon, M.D., Ph.D., \\ Michael Muhonen, M.D., Daniel Shrey, M.D.
}

\section{Introduction}

Invasive intracranial monitoring using subdural grids has long been used for language and motor mapping as well as seizure onset zone localization in epilepsy surgery patients. During extraoperative functional mapping, direct cortical electrical stimulation is sequentially given to determine whether the stimulated cortical surface results in a change in neurologic function. If a neurological response is seen, then that cortical tissue is thought to correspond to that particular function. Standard grid contacts are spaced $1 \mathrm{~cm}$ apart.

\section{Methods}

We describe the use of a high-density, $29 \mathrm{~mm} \times 29 \mathrm{~mm}$ subdural grid (Ad-Tech FG64C-MP03X000) with 64 platinum contacts arranged in an $8 \times 8$ configuration with $3 \mathrm{~mm}$ on-center interelectrode spacing to localize the seizure onset zone and map language and motor function in a 15-year-old girl with a lesion abutting eloquent cortex. We outline charge density, safety considerations as well as stimulation strategies employed during the mapping process.

\section{Results}

Clinical interpretation of the long-term subdural EEG data was unaffected by the smaller electrode size and closer interelectrode distances. The seizure onset zone was localized to a smaller region using the high-density grid than would have been possible with a standard grid. During mapping, facial and hand motor regions were activated with stimulation currents of $0.5 \mathrm{~mA}$ and $2 \mathrm{~mA}$, respectively. The high-density grid did not encompass the language cortex. However, an adjacent standard grid localized language function with speech arrest observed with $2 \mathrm{~mA}$ of stimulation. The epileptogenic focus was subsequently resected, and she had an Engel Class 1B outcome at two months post-resection. She did not sustain any complications from cortical stimulation using the high-density grid.

\section{Conclusion}

High-density grids can be used safely and more precisely to localize the seizure onset zone, as well as test for language and motor function in pediatric patients undergoing invasive intracranial monitoring. 


\section{Prenatal Closure of Myelomeningoceles Reduces Chiari-associated Brainstem Dysfunction}

Paul A. Grabb, M.D.; Paige Lundy, M.D.; Michael D. Partington, M.D.; Emanuel Vlastos, M.D.

\section{Objective}

Prenatal closure of myelomeningoceles reduces anatomic severity of Chiari II malformations compared to postnatal closure. Correlating anatomic change with functional outcome, however, has not been shown. Symptom frequency ascribed to the brainstem has not been thought to differ between groups. Symptom severity between groups however, has not been fully addressed.

\section{Material-Methods}

We reviewed imaging and records for gestational age, Chiari malformation anatomic grade (normal, mild, moderate, severe), significant brainstem dysfunction (SBD), ventricular size, and myelomeningocele level in all neonates repaired either prenatally $(n=13)$ or postnatally $(n=45)$ over four years. SBD was defined as neurogenic dysphagia requiring gastrostomy, airway protection and respiratory insufficiency requiring tracheostomy, vocal cord or glottic surgery, or repeated admissions requiring intubation.

\section{Results}

Twelve neonates (27\%) in the postnatal group had SBD within the first month of life. Of these 12, two had "mild", six had "moderate", and four had "severe" anatomic grades of Chiari malformations. Two underwent Chiari decompressions without improvement. None with prenatal closure experienced SBD, or anatomically had moderate or severe Chiari malformations. Shunts were placed in $64 \%$ and $8 \%$ of the postnatal and prenatal groups, respectively. Endoscopic techniques were employed successfully in four infants, two in each group. Ventricular sizes at birth were a median of $16 \mathrm{~mm}$ and $19 \mathrm{~mm}$ for prenatally and postnatally treated neonates, respectively. Median gestational ages were 36.6 and 38 weeks, for prenatally and postnatally treated neonates, respectively. Median and mean functional myelomeningocele levels were L4 for both groups.

\section{Conclusion}

Despite published autopsy findings of dysplastic brainstem nuclei and pathways in some children with spina bifida, and more recent publications that broadly address any apnea as not favorably influenced by prenatal repair, we found prenatal repair is associated with marked reduction in frequency of SBD traditionally ascribed to brainstem compression or dysplastic brainstem in these neonates. 


\title{
The Hydrodynamic Properties of Commonly Used Ventriculoperitoneal Shunts
}

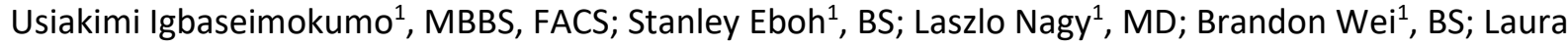 \\ Arnold-Perez ${ }^{1}$, BS; Roy Jacob ${ }^{1}$, MD \\ ${ }^{1}$ Texas Tech University Health Sciences Center School of Medicine, Lubbock, Texas
}

\section{Introduction}

Cerebrospinal fluid shunt valve technology has evolved but very little over the last half century. Today surgeon preference is still the main factor in deciding on a valve. Current experience suggests that up to $40 \%$ of CSF shunts may fail within the first year. There is widespread consensus that over-drainage occasioned by the siphon effect in the erect position is a significant contributing factor. Opinions differ widely amongst practitioners, and there is a dearth of information on the properties of these shunts. The primary research question therefore was "is there a closing pressure with most of the commonly used valves with or without an anti-siphon mechanism?"

\section{Materials and Methods}

Four commonly used CSF shunt valves were examined in vitro with Pressure-Flow Experiments at room temperature under atmospheric pressure. The ProGAV ${ }^{\circledast}$ valve with Shuntassistant ${ }^{\circledR}$ was selected as an example of a valve with anti-siphon mechanism. The Codman Precision ${ }^{\circledR}$ valve and Integra ${ }^{\circledR}$ DP $^{\mathrm{Tm}}$ differential pressure valves (low pressure and high-pressure valve) were selected as examples of valves without anti-siphon mechanism. The experimental set up consisted of a manometer, $55 \mathrm{~cm}$ tall, filled with water and connected to the valve being tested by a three-way tap. A standard amount of distal tubing $80 \mathrm{~cm}$ was then connected to the valve. The flow rates were measured with the distal tubing at 90 degrees, 135 degrees and 180 degrees in relation to the manometer. The 180 degrees simply means manometer valve and distal tubing were in a vertical line. Once the three-way tap was opened, the time taken to drain from $55 \mathrm{~cm} \mathrm{H} 2 \mathrm{O}$ to zero was recorded by two independent observers. This was repeated ten times and the average for the two observers was used for the calculations below. To examine the effect of pressure on the flow rate, the time taken to drain from 55 to $35 \mathrm{~cm}$ and from 35 to $15 \mathrm{~cm}$ was also recorded and compared for each valve.

\section{Results}

In the 180 degrees position all valves drained to zero with no closing pressure. The mean flow rate in the 180 degrees (erect position) for the Integra low pressure valve was $36 \mathrm{cc}$ per min and the mean flow rate for the high pressure-valve was $32.4 \mathrm{cc}$ per $\mathrm{min}$, with both valves draining to zero. At 90 degrees, the Integra low-pressure valve drained at flow rate of $12.6 \mathrm{cc}$ per min in the pressure range 55 to 35 $\mathrm{cmH} 2 \mathrm{O}$ and $6.0 \mathrm{cc}$ per min (a drop of 51\%) in the pressure range 35 to $15 \mathrm{~cm} \mathrm{H} 2 \mathrm{O}$. The respective figures for the high-pressure valve were a flow rate of $7.8 \mathrm{cc}$ per min in the 55 to $35 \mathrm{cmH} 2 \mathrm{O}$ pressure range and $2.4 \mathrm{cc}$ per min in the 35 to $15 \mathrm{cmH} 20$ pressure range.

The ProGAV valve set at $10 \mathrm{~cm}$ with ShuntAssistant ${ }^{\circledR}$ of $10 \mathrm{~cm}$ was used for these measurements. The mean flow rate at 180 degrees was $16.8 \mathrm{cc}$ per min. It also drained to zero with no closing pressure in 
the vertical (erect) position. The flow rate in the pressure range 55 to $35 \mathrm{~cm} \mathrm{H} 20$ was $6.6 \mathrm{cc}$ per min and in the 35 to $15 \mathrm{cmH} 20$ pressure range was $3.6 \mathrm{cc}$ per min. The flow rate for the Codman Hakim Precision valve (low pressure) in the 180 degrees (the erect) position was $27.6 \mathrm{cc}$ per min. Over the pressure range 55 to $35 \mathrm{~cm} \mathrm{H} 2 \mathrm{O}$, the flow rate was $10.8 \mathrm{cc}$ per min and in the pressure range 35 to 15 $\mathrm{cmH} 2 \mathrm{O}$ it was $4.2 \mathrm{cc}$ per min.

\section{Conclusion}

This set of Pressure - Flow experiments demonstrated that there was no closing pressure in the tested valves in vitro, indicating that the siphon effect, effectively overrides the combined resistance of the valve and the antisiphon mechanism in the ProGAV valve. All the valves tested show a predictably higher flow at the higher-pressure range of 55 to $35 \mathrm{cmH} 2 \mathrm{O}$ compared to the lower pressure range of 35 to $15 \mathrm{~cm} \mathrm{H} 2 \mathrm{O}$.

All the authors have no conflicts of interest to declare. 


\section{Ventricular-peritoneal Shunt Overdrainage is Driven by Gravity and Cerebrospinal Fluid Pulsations: Computer Simulation and Benchtop Testing}

David A. Hsu MD PhD'; Stephanie A. Armstrong, MS²; Joyce Koueik, MD, MS²; Mark Kraemer, $\mathrm{MD}^{2}$; Zhe Yang, $\mathrm{MS}^{3}$; Jessica Meah, $\mathrm{BS}^{1}$; Christopher Luzzio, $\mathrm{MD}^{1,3}$, and Bermans J. Iskandar, $M D^{2}$

Departments of Neurology ${ }^{1}$, Neurological Surgery ${ }^{2}$, and Engineering ${ }^{3}$, University of Wisconsin, Madison, WI

While gravity effects are known to drive CSF shunts to overdrain, valves designed to counteract gravity effects (anti-siphon, flow-regulated, and programmable valves) are often ineffective at preventing overdrainage. Via computer simulations and benchtop testing presented here, we show that ICP pulsations induced by Valsalva maneuvers and even cardiac pulsations can increase CSF drainage. The clinical significance is that prevention of CSF overdrainage will require a valve system that not only mitigates the gravity effect, but also counteracts the pulsation effects. In light of mounting evidence suggesting that chronic shunt overdrainage may drive proximal shunt obstruction, these results would impact efforts to improve shunt function and survival.

None of the authors has any conflict to disclose. 


\title{
Reliability of the Radiopharmaceutical Shunt Flow Study in the Presence of Stable Ventricular Size for the Detection of a Cerebrospinal Fluid Shunt Malfunction
}

\author{
Main Author: \\ J. Gordon McComb, MD ${ }^{1,2}$ \\ GMcComb@chla.usc.edu \\ Co-author: \\ Jeffrey J. Quezada, BS ${ }^{1}$ \\ jquezada@chla.usc.edu \\ ${ }^{1}$ Division of Neurosurgery, Children's Hospital Los Angeles, Los Angeles-CA, USA \\ ${ }^{2}$ Department of Neurological Surgery, Keck School of Medicine, University of Southern California, Los Angeles-CA, \\ USA
}

Topic

Clinical Research

\section{Disclosure information}

There were no conflicts of interest for either author in the conduct of this research or writing of the abstract.

\section{Objective}

To determine the reliability of a radiopharmaceutical (RP) shunt flow study in the presence of stable ventricular size for the detection of a cerebrospinal fluid (CSF) diverting shunt malfunction.

\section{Methods}

After obtaining IRB approval all CSF RP shunt flow studies done between January 1, 2014 and January 1, 2019 were identified. Included in the study were only those patients in whom a $\mathrm{MRI} / \mathrm{CT}$ scan was done during the hospital admission for shunt malfunction and showed no increase in ventricular size when compared with the most recent prior MRI/CT scan when the patient was asymptomatic. The patient's age, sex, etiology of the hydrocephalus, shunt distal site, non-programmable versus programmable valve, operative findings if the shunt was revised and a follow-up for a minimum of 90 days after admission were recorded. The RP shunt flow study consisted of tapping the reservoir and injecting Tc-99m DTPH as per a set protocol. The RP shunt flow study was considered normal if there was rapid reflux of tracer into the ventricles followed by rapid distal tracer flow from the distal end of the shunt. No ventricular reflux was considered a proximal malfunction while very slow, no distal runoff, or extravasation of tracer along the shunt tract was reported to be a distal obstruction. 


\section{Results}

Identified were 142 RP flow studies in 112 patients meeting the above criteria. Of the 112 normal flow studies operative intervention was not undertaken in 102 (91\%). Of the 10 (9\%) operated patients eight had a proximal obstruction, 1 distal and 1 no obstruction. Of the 30 abnormal flow studies 9 (30\%) patients were not operated on as their symptoms of shunt malfunction subsided. Of the 21 (70\%) operated patients obstruction was proximal in 9, distal in 5 and for 7 the shunt tubing was fractured or disconnected. Regression analysis indicated a significant association between the flow study interpretation and the odds for shunt revision $(\mathrm{OR}=27,95 \% \mathrm{Cl}=10-75, \mathrm{p}<0.0001)$. No other clinical variables were significant. The shunt flow study alone compared with the shunt flow study plus MRI/CT did not change the sensitivity $70 \% / 70 \%$, but did increase the specificity from $92 \%$ to $100 \%$ and the accuracy from 87 to $94 \%$.

\section{Conclusion}

RP shunt flow studies in patients with symptoms of shunt malfunction in whom no change in ventricular size occurred when non-symptomatic was of definite value in deciding as to operatively intervene. 


\title{
Corpus Callosotomy Performed with Laser Interstitial Therapy -
}

\section{An Initial Experience}

\author{
Roland, Jarod L. and Smyth, Matthew D.
}

\section{Introduction}

Corpus callosotomy is a palliative procedure that can decrease the severity of seizures in select patients with medically refractory epilepsy, particularly atonic seizures. Laser interstitial thermal therapy (LITT) is a minimally invasive stereotactic technique for ablating brain tissue. A common barrier to referral to epilepsy surgery is patient/family and neurologists' concerns surrounding traditional invasive surgery. LITT for callosotomy is a less invasive option that has the potential for successful treatment while minimizing morbidity.

\section{Methods}

We reviewed our cases of LITT for corpus callosotomy by collecting patient demographics, procedure notes, complications, and outcomes from chart records. We also analyzed pre and post procedure magnetic resonance imaging to determine the extent of callosum ablated. Advanced imaging with resting state functional connectivity was analyzed in selected cases to evaluate effective disconnection.

\section{Results}

We performed 11 procedures in 10 patients. Five patients received an anterior two-thirds LITT callosotomy as their first procedure. One complete ablation for a partial callosal agenesis, three posterior completions after prior anterior callosotomy, one to complete the basal frontal disconnection of a remnant at the rostrum after hemispherotomy was performed, and one for a focal ablation of the splenium in a patient with a light-sensitive epilepsy syndrome. One patient experienced a supplementary motor area syndrome that completely resolved. The ROSA robotic system was used to place 1-3 fibers per case. Inpatient length-of-stay ranged from 1-6 days (median 2). Seizure control was comparable to traditional microsurgical disconnection.

\section{Conclusion}

LITT is a promising minimally invasive option for corpus callosotomy. Scenarios such as completion of prior partial disconnection and partial agenesis of the corpus callosum are excellent candidates for LITT. However, unfavorable morphology such as thin and high arching corpus callosum may be better treated by open procedures. More cases and longer follow up are needed to better stratify the risk-benefit profile.

\section{Disclosures}

JLR - None; MDS - None 


\title{
Early Intervention: The Use of a Simple Home Care Program in the Post - Craniotomy Patient to Reduce Readmission and Lower the Overall Cost of Care
}

\author{
Brian D Rothstein MD/MS, Megan Louttit MPH
}

As healthcare continues to evolve, professionals seek to identify more effective and efficient tools to better care for our patients. Readmissions continues to represent a significant portion of the sky-rocketing costs in healthcare. With the implementation of the Medicare 30-day rule, many institutions have been scrambling to find sufficient programs to reduce readmissions. Through systematic review, we observed a $22 \%$ readmission rate for adult patients undergoing craniotomy in 2014 , of which, $52.6 \%$ were patients who were discharged to home. We also noted that $49.5 \%$ of our readmissions occurred within 14 days of discharge. At a cost of approximately $\$ 50,000.00$ per readmission, this was identified as a key area of concern. We developed a basic program for patients discharged home, based on receiving education and an evaluation by a Home Care nurse within $48 \mathrm{hrs}$ of discharge. We education the team of Home Care nurses on the key aspects of post-craniotomy care and asked them to evaluate the safety of the home, as well as review discharge and medication instructions. The cost of a single Home Care encounter is approximately $\$ 125.00$. Using this system, we were able to reduce our readmission rate for patients discharged to home from approximately $20 \%$ in 2015 , to $11.9 \%$ in 2016 and $10.2 \%$ in 2017. The total cost of all the Home Care services per year received by this cohort of patients was equivalent to one readmission. Our results indicate that successful, early intervention at home with an appropriately trained Home Care nurse, has the potential to dramatically reduce readmissions in post-craniotomy patients. We now look to apply these same principles to our pediatric population. 


\title{
Feasibility of Using Computational MRI to Identify Epileptic Foci in Children
}

\author{
Heather S. Spader, $\mathrm{MD}^{1}$, Jonathan O'Muircheartaigh, $\mathrm{PhD}^{2}$, Pratik A. Talati, MD, $\mathrm{PhD}^{3}$, \\ Scellig Stone, MD, PhD ${ }^{4}$, Sanjay P. Prabhu, MBBS, FRCR ${ }^{5}$
}

1. Department of Pediatric Neuroscience, Joe DiMaggio Children's Hospital, Hollywood, FL

2. Department of Forensic Neurodevelopmental Science and Perinatal Imaging and Health, Kings College, London, UK

3. Department of Neurosurgery, Massachusetts General Hospital, Boston, MA

4. Department of Neurosurgery, Boston Children's Hospital, Boston, MA

5. Department of Radiology, Boston Children's Hospital, Boston, MA

Identifying surgical targets in children with drug resistant epilepsy can be challenging. Compared to adults, focal cortical abnormalities are more common and exhibit greater spatial variability. This variability makes radiological identification of potentially epileptogenic tissue abnormalities challenging. To address this, we used computational MRI to try to identify pathologically defined tissue abnormalities in a retrospective case series of children with focal epilepsy who subsequently had surgical resection.

We collected T2-weighted FLAIR MRI scans from a cohort of 36 children ( 29 with focal epilepsy and 8 controls) across a wide age range ( 6 months to 18 years). 7 patients in this cohort had MRI-negative epilepsy.

After registering images non-linearly to a common space, we applied an outlier detection algorithm to investigate tissue abnormalities as multivariate outliers from the normal range of the other datasets. The "normal" range included children with epilepsy so this method assumes marked heterogeneity in the spatial location and extent of epileptic tissue across subjects.

Spatially variable radiological signs (local atrophy, asymmetry, transmantle signs, tissue hyperintensity) were detectable in 18 of 29 (62\%) patients with FLAIR MRI abnormalities. Of the MRI negative subjects, the model predicted epilepsy foci in 4/7 $(57 \%)$ of these subjects.

This preliminary study utilized computational neuroimaging to create a model from MRI scans obtained primarily from patients with epilepsy in order to detect outliers to make predictions about the location of epileptic foci. Preliminary data show a correlation between the model and epileptic foci in around $57-62 \%$ of subjects. This approach does not aim to classify pathological tissue but shows promise in its ability to provide an automated, quantitative, and rapid screening test for interpretation alongside standard clinical imaging and clinical data. 
(a) MRI Reported Negative Right Frontal Resection

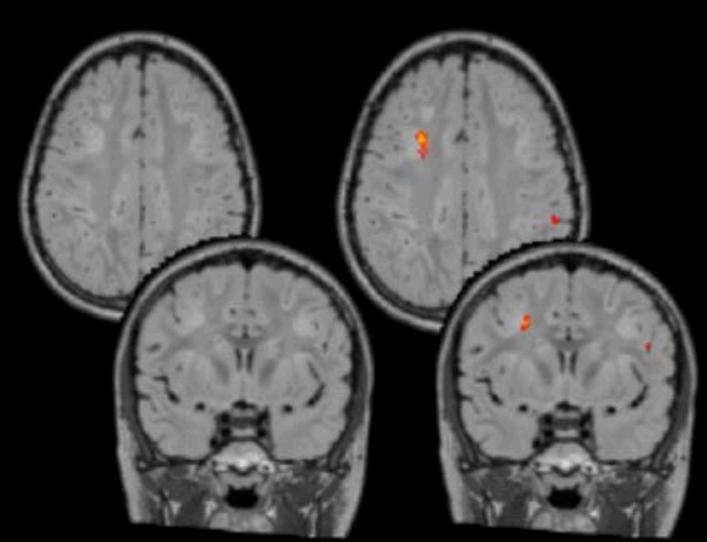

(b) MRI Reported Positive Left Resection

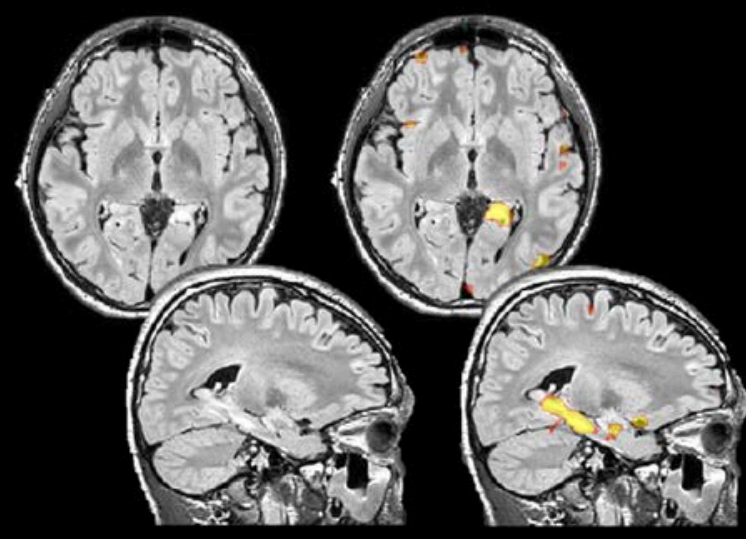


$43^{\text {rd }}$ Annual Meeting

The American Society of Pediatric Neurosurgeons

Jointly provided by AANS

\title{
Craniosynostosis is a Concern for Patients with Chiari and Idiopathic Intracranial Hypertension and Misdiagnosis can Lead to Incorrect Treatment of the Patient
}

\author{
Ashley G. Tian, MD \\ Department of Neurosurgery, The University of Arizona, Cardon Children's Hospital, Mesa, AZ
}

It is well known that Chiari I malformation can be associated with craniosynostosis and several recent studies have looked at the incidence of Chiari malformation associated with craniosynostosis and craniosynostosis mimicking idiopathic intracranial hypertension. Misdiagnosis of these syndromes may lead to inappropriate management.

Seven patients were identified over the last 5 years with unusual presentation of neurosurgical problems, which were associated with craniosynostosis. Not all patients required interventions. The most common presentation was sagittal synostosis with Chiari, which is well documented in the literature. Two patients had Chiari decompressions performed by two previous neurosurgeons for headaches and were subsequently diagnosed with sagittal synostosis on follow up. One underwent a subsequent cranial vault expansion with improvement in headaches and the other had ICP monitoring with normal ICP and no further intervention. One was diagnosed as an infant with sagittal synostosis and had a vault expansion, despite a normal cranial index, due to the presence of a Chiari. A 2yo had a Chiari and sagittal synostosis diagnosed on work up for seizures and is being observed. One presented with back pain and was found to have sagittal synostosis after an extensive work up. Back pain and increased ICPs resolved with vault expansion. A 6yo presented with papilledema and appeared proptotic. Cl was 0.87 . CTH showed pansynostosis. "Pseudotumor" resolved with vault expansion. One presented with Chiari and significant brainstem edema and was found to have multisutural synostosis. This resolved with cranial vault expansion.

Careful review of imaging and physical findings in patients with Chiari or other common neurosurgical entities may lead to a more accurate diagnosis and better care. 
$43^{\text {rd }}$ Annual Meeting

The American Society of Pediatric Neurosurgeons

Jointly provided by AANS

\section{Identification of New Chromosomal Abnormality in a Familial X- linked Congenital Hydrocephalus Pedigree}

Tong Yang MD PhD, Sanford Brain and Spine Fargo, Pediatric Neurosurgery

\section{Background}

Hydrocephalus affects $\sim 1.1$ per 1,000 infants. The development of hydrocephalus is a heterogeneous disease process with various causes. Knowledge is limited about the genetic pathway(s) underlying the development of hydrocephalus in human patients. Because various clinical conditions can be associated with hydrocephalus development, multiple genes are likely involved in the disease process. X-linked L1 gene mutations have been identified as causes of congenital hydrocephalus. More recently other autosomal genes including MPDZ, CCDC88C, TRIM71, SMARCC1, PTCH1, and SHH, were identified to be linked to congenital hydrocephalus.

\section{Result}

We have identified a family with two brothers affected by congenital hydrocephalus. Both needed surgical treatment of the condition. There is a family history of congenital hydrocephalus on the mother's side. Both parents do not have hydrocephalus and there are additional non-hydrocephalic children (of both gender) in the family. Chromosomal microarray analyses have identified two regions of duplications on the $X$ chromosome: a $294 \mathrm{~kb}$ segment at Xq26.3 and a 369kb segment at Xq27.1. Known genes within the two regions have not been previously linked to hydrocephalus in humans. One known gene (GPR101, OMIM\# 300393) within Xq26.3 has been linked to X-linked acrogigantism; and one known gene (SOX3, OMIM\# 313430) within Xq27.1 has been observed in X-linked hypopituitarism, infundibular hypoplasia and neural tube defect. The brothers have identical chromosomal changes suggesting a direct inheritance of the genetic aberrance from the mother. Currently we are seeking funding opportunities to perform whole genome DNA sequencing of the pedigree to identify molecular changes that are linked to the congenital hydrocephalus in this family.

\section{Conclusion}

If new genes or genetic changes are identified within the two duplication regions that are linked to this family with congenital hydrocephalus, we will be able to discover another previously unknown player within the pathogenesis of congenital hydrocephalus. 


\title{
Long Term Outcome of Rhizotomy for the Management of Childhood Spasticity- Functional Improvement and Complications
}

\author{
Albert Tu MD FRCSC FAANS ${ }^{1}$ and Paul Steinbok MBBS FRCSC ${ }^{2}$ \\ ${ }^{1}$ Division of Pediatric Neurosurgery, Children's Hospital of Eastern Ontario \\ ${ }^{2}$ Division of Pediatric Neurosurgery, Children's Hospital of British Columbia
}

\section{Background}

Selective Dorsal Rhizotomy (SDR) for the management of lower extremity spasticity is a surgical technique that has existed since the 1900s. While much evidence supports its efficaciousness in reducing tone in the short term, limited information exists detailing the long term outcome and evolution over time of patients undergoing SDR.

\section{Methods}

All publications with 10 years or more of outcome data on patients undergoing SDR were identified from Medline and Embase databases using the search term 'Rhizotmy'. Only publications that were in English, included patients with cerebral palsy under the age of 21, and discussed SDR for lower extremity spasticity were included. Case reports, reviews without primary data, or publications not accessible online were excluded from review.

\section{Results}

A total of 2128 publications were initially identified, of which 19 papers describing 1054 patients fit inclusion and exclusion criteria. GMFCS in most patients improved or remained stable over time after surgery, although durability of improvement and final outcome was dependant on initial functional status. Tone was noted to also substantially improve although a small proportion required additional oral or injectable pharmacologic agents, and an even smaller proportion required baclofen pumps. Upper extremity function was also noted to improve substantially after SDR. A significant number of patients were independent for activities of daily living and were engaged in secondary education or gainful employment. A significant proportion of patients still required orthopedic surgery to the lower extremities after SDR, although the incidence of spinal deformity was not observed to be substantially elevated over that of natural history.

\section{Conclusion}

SDR offers substantial improvements to a number of domains beyond pure tone reduction. These changes are durable over time, although patient selection is crucial in identifying those patients that will have the most benefit. Long term follow up is in important in this population given the potential need for further interventions that still exist in many patients. 


\title{
Correspondence and reprint requests
}

\author{
Albert Tu, MD FRCSC FAANS \\ Division of Pediatric Neurosurgery \\ Children's Hospital of Eastern Ontario \\ Clinic C-9, Neurosurgery, CHEO \\ 401 Smyth Rd, Ottawa ON \\ K1H 8L1, Canada
}

Ph: 6137377600 ext 3358

Fa: 6137384879

Email: atu@cheo.on.ca

\section{Keywords}

Rhizotomy, outcome, long term, spasticity, pediatric, cerebral palsy, review, complications

\section{Disclosures}

The authors have no personal, financial, or institutional interest in any of the drugs, materials, or devices described in this article. 


\title{
The Impact of Resident Work-related Factors on Risk for Burnout:
}

\section{A Global Neurosurgery Pilot Study}

\author{
Hasan Syed, MD
}

\section{Objective}

This study aims to examine the risk for burnout in neurosurgery trainees across the globe, to compare some work-related factors that may contribute to it, and to determine if there are international and intercultural differences.

\section{Methods}

A 16-question survey was designed to generate sufficient responses for analysis, and request to complete the online survey was then broadcasted through social media networks of global neurosurgeons. The first half of the survey examined the work-related factors that may contribute to burnout. The second half, adopted from the Maslach survey, studied the respondents' attitude and emotional responses towards their training, patients and work environment. Responses were anonymized before analysis. The answers to the "burnout questions," collected on a 5-point Likert-type scale, were converted to numerical values for statistical examination and comparisons.

\section{Results}

There were 797 responses to the survey from 93 countries, and 243 of those were from countries designated as "low-to-middle income" (LMIC). Amongst the residents and fellows who responded to all six "burnout questions," $20.7 \%$ of the respondents scored in the range designated "at risk for burnout." Logistic regression analysis showed that operative case load, frequency of on-call duty, and total work-hour were drivers for burnout in the global cohort, but the relationship between case load and burnout risk was inverse. Trainees with fewer than 100 cases were at significantly higher risk for burnout compared to those with more robust operative exposure.

Intercontinental comparisons revealed that trainees in the United States and Canada had the lowest proportion at risk for burnout (11.2\%), whereas the Europeans had the highest (26.9\%). Considering that, in general, European trainees work fewer hours and have less on-call duties than others, these results may be related to the eroding case load as perpetrated by overstringent restriction on work hours in Europe. Conversely, trainees in LMIC work more than their international colleague, but their average total burnout score and proportion at risk for burnout were identical by global comparison, pointing to a resilience, the origins of which this pilot study was not equipped to examine.

\section{Conclusion}

Risk for burnout in neurosurgery residents and fellows are driven by multiple factors from personal, demographic, programmatic to institutional. Among work-related factors, long and frequent shifts were found to contribute to the risk of burnout in the global cohort. However, the impact of these factors was not uniform across the globe. Despite tough restriction on work hours, but perhaps because of them, Europeans had the highest risk for burnout. In contrast, LMIC trainees were comparatively immune to burnout driven by long hours and frequent overnight shifts. 


\title{
Non-opioid Pain Control following Chiari Malformation Surgery in Children:
}

\section{An Initial Experience with Injectable Liposomal Bupivacaine (Exparel)}

\author{
Edward S. Ahn¹, Victor M. Lu¹, David J. Daniels ${ }^{1}$, Dawit T. Haile ${ }^{2}$ \\ 1. Department of Neurosurgery, Mayo Clinic, Rochester, MN, US. \\ 2. Department of Anesthesiology, Mayo Clinic, Rochester, MN, US.
}

\section{Background}

The surgical management of Chiari malformation type 1 (CM-1) in children is known to include a painful postoperative course during hospitalization. Due to reliance concerns, attempts to minimize opioid use in this demographic are preferable. We report our initial experience using Exparel, a single-dose intraoperative intramuscular injection of liposomal bupivacaine with CM1 decompression.

\section{Methods}

We conducted a retrospective review of all CM-I surgeries performed in children ( $<18$ years) at the Mayo Clinic, Rochester between 2018-2019 that were administered intramuscular Exparel during the operation. Outcomes of interest were postoperative opioid (oxycodone or hydromorphine) use, pain scores during hospitalization graded by visual analog scale $(0-10,10$ being worst pain possible), and length of stay.

\section{Result}

Four female and three male patients diagnosed with CM-I and treated by surgery with Exparel satisfied our selection criteria, with median age 14 years (range, 12-17). During hospitalization, 5 patients required two days with opioid administration with median dose $30 \mathrm{mg} /$ day (range, 5$70 \mathrm{mg}$ ), and 2 patients requiring no opioid doses at all during hospitalization. Throughout hospitalization, median overall pain score reported was 0 (range, $0-7$ ), with pain peaking at median of 4 (range, 0-9). Median overall length of stay was 3 days (range, 2-5). These outcomes compared favorably to a historic control cohort of CM-I patients that did not receive Exparel with respect to pain scores and opioid requirements.

Conclusions: The use of Exparel in the setting of CM-I surgery results in favorable pain control in children during hospitalization, which may be superior to that of opioid-based management. Future studies are required to validate these findings in larger cohorts.

Word count: 260 


\title{
Natural History of Pediatric Chiari Type 1 Malformation: A Retrospective Analysis
}

\author{
Matthew Carey ${ }^{1}$, William Fuell BS ${ }^{1}$, Gregory W. Albert MD ${ }^{1,2}$ \\ ${ }^{1}$ Division of Neurosurgery, Arkansas Children's Hospital, Little Rock, AR \\ ${ }^{2}$ Department of Neurosurgery, University of Arkansas for Medical Sciences, Little Rock, AR
}

\section{Introduction}

Chiari I Malformation (CM-I) is often diagnosed incidentally. While asymptomatic CM-I is generally considered to be a benign condition, reports on the natural history are limited. In this study, we seek to document the natural history of CM-I in a large cohort of patients and identify risk factors for progression over time.

\section{Methods}

$330 \mathrm{CM}-\mathrm{I}$ patients were divided into three groups: surgery within 6 months post-diagnosis, surgery over 6 months post-diagnosis, and never having surgery. From the database, characteristics such as gender, race, age at diagnosis, signs/symptoms, and MRI imaging results were compared.

\section{Results}

30 patients had surgery within 6 months of diagnosis (G1), 18 had surgery after 6 months (G2), and the remaining 282 did not have surgery (G3). For $\mathrm{G} 2$, the time post-diagnosis for surgery was approximately 15 months. Between $\mathrm{G} 2$ and $\mathrm{G} 3$, there was no observable difference in tonsillar descent, tonsillar impaction at the foramen magnum, gender, race, or associated conditions such as tethered cord, neurofibromatosis, migraines, or seizures. However, G2 demonstrated increased incidence of syringomyelia ( $33 \%$ vs $14 \%$ ), Valsalva headaches ( $22 \%$ vs $10 \%)$, prematurity ( $11 \%$ vs $4 \%$ ), and developmental delay ( $28 \%$ vs $13 \%$ ).

\section{Conclusions}

The data show that the majority of patients diagnosed with CM-I do not require surgical intervention. Additionally, there are only a small number of patients that did not initially have surgery that required later intervention. The results indicate that, of initially asymptomatic patients, individuals with Valsalva headaches, a syrinx, premature birth, or developmental delay may be at increased risk for eventually requiring surgical intervention. This information is useful to pediatric neurosurgeons in counseling patients and families with a new diagnosis of CM-I.

\section{Disclosures}

This work is supported by a University of Arkansas for Medical Sciences Research Scholar Pilot Grant Award in Child Health. The authors have no conflicts of interest to disclose. 


\title{
Surgical Correction of Cervical Kyphosis in Diastrophic Dysplasia with Anterior Corpectomy, Posterior Tethering with $360^{\circ}$ Fusion LOE-Case Series Level IV
}

\author{
John A Heydemann, MD, Kenneth J Rogers, PhD, Jeffrey W Campbell, MD, \\ Suken A Shah, MD, and William G MacKenzie, MD
}

\section{Background}

Diastrophic dysplasia is caused by mutations in the diastrophic dysplasia sulfate transporter gene. Cervical kyphosis, kyphoscoliosis, spina bifida oculta, and hyperlordosis of the lumbar spine are common. The natural history of cervical spine kyphosis is spontaneous resolution, yet occasionally it does not, resulting in neurologic symptoms and severe deformity. We describe the patient characteristics pre- and post-surgery for anterior corpectomy with cage implantation and posterior tethering compared to a matched control of posterior only fusion technique.

\section{Methods}

This is a retrospective study of patients with diastrophic dysplasia who underwent cervical spine surgery. These patients underwent either anterior corpectomy and $360^{\circ}$ fusion or posterior fusion alone. All patients were treated at a single institution and by one surgeon. Indications for surgery included cord compression with or without myelomalacia, cervical instability and severe or progressive kyphosis. Six cases of cervical kyphosis were evaluated by measuring the $\mathrm{C} 2-\mathrm{C} 7 \mathrm{Cobb}$ angles and $\mathrm{T} 1$ slope on lateral neutral cervical radiographs prior to surgery and at the last know post-op follow up. Neurologic examinations both pre- and postop, blood loss, and complications such as pseudoarthrosis were also reviewed.

\section{Results (see Tables)}

A total of 47 patients with diastrophic dysplasia were collected from 1984 to 2015. Nineteen patients were followed for cervical kyphosis and six of those patients required surgery. The average age at the time of surgery in the anterior group was 40 months and in the posterior group 21 months. Two of the 4 in the anterior group had cord compression with myelomalacia seen on flexion and extension magnetic resonance imaging. Two patients with progressive kyphosis were treated with posterior tethering only to allow for anterior growth. The mean estimated blood loss was $294 \mathrm{~mL}$ anteriorly and $75 \mathrm{~mL}$ posteriorly. All patients were noted to have a complete fusion. One patient sustained a dural tear, which was successfully repaired. This patient also developed erythema at the halo pin-sites treated with antibiotics to resolution. The mean starting Cobb angle was 90 degrees of kyphosis in the anterior group and 
70 degrees of kyphosis in the posterior group. Post-operatively or at follow-up, the mean for the anterior group was 20 degrees of kyphosis and 23 degrees of lordosis in the posterior group. Mean pre-operative T1 slope in the anterior group was 10 degrees of lordosis and 8 degrees of kyphosis in the posterior group. This changed to 17 degrees and 28 degrees of kyphosis in the respective groups.

\section{Conclusion}

Spontaneous resolution is common in children with cervical kyphosis in diastrophic dysplasia.

There exists a subset of patients with stiff, progressive, severe curves who develop cord compression and myelomalacia. Posterior surgery will not address the cord compromise. This can be more effectively managed with anterior decompression along with anterior and posterior fusion to improve overall cervical alignment and prevent further cord damage.

\section{Key Words: Diastrophic dysplasia, Cervical kyphosis}

Table 1: Demographics and Clinical Characteristics

\begin{tabular}{|c|c|c|c|c|c|c|c|c|c|}
\hline $\begin{array}{l}\text { Subject } \\
\text { ID }\end{array}$ & Procedure & Gender & $\begin{array}{l}\text { Age at } \\
\text { surgery } \\
\text { (mo) }\end{array}$ & $\begin{array}{l}\text { Indication for } \\
\text { Surgery }\end{array}$ & $\begin{array}{l}\text { EBL } \\
(\mathrm{mL})\end{array}$ & $\begin{array}{l}\text { Transfusion } \\
(\mathrm{mL})\end{array}$ & $\begin{array}{l}\text { Halo Post- } \\
\text { operatively }\end{array}$ & Complications & Outcome \\
\hline 1 & $\mathrm{~A} / \mathrm{P}$ & $\mathrm{F}$ & 56 & $\begin{array}{l}\text { Cord } \\
\text { compression } \\
\text { with } \\
\text { myelomalacia }\end{array}$ & 250 & 130 & $\mathrm{Y}$ & None & $\begin{array}{l}\text { Healed } \\
\text { fusion }\end{array}$ \\
\hline 2 & $\mathrm{~A} / \mathrm{P}$ & M & 24 & $\begin{array}{l}\text { Cord } \\
\text { compression }\end{array}$ & 150 & 189 & $\mathrm{Y}$ & None & $\begin{array}{l}\text { Healed } \\
\text { fusion }\end{array}$ \\
\hline 3 & $\mathrm{~A} / \mathrm{P}$ & M & 39 & $\begin{array}{l}\text { Cord } \\
\text { compression } \\
\text { with } \\
\text { myelomalacia }\end{array}$ & 275 & 160 & $\mathrm{Y}$ & $\begin{array}{l}\text { Dural tear } \\
\text { during } \\
\text { posterior } \\
\text { portion. } \\
\text { Erythematous } \\
\text { halo pin-sites }\end{array}$ & $\begin{array}{l}\text { Healed } \\
\text { fusion }\end{array}$ \\
\hline 4 & $\mathrm{~A} / \mathrm{P}$ & M & 41 & $\begin{array}{l}\text { Cord } \\
\text { compression }\end{array}$ & 500 & 297 & $\mathrm{Y}$ & None & $\begin{array}{l}\text { Healed } \\
\text { fusion }\end{array}$ \\
\hline 5 & $\mathrm{P}$ & $\mathrm{F}$ & 13 & $\begin{array}{l}\text { Cervical } \\
\text { instability C3- } \\
\text { C4 }\end{array}$ & 50 & 0 & $\mathrm{Y}$ & None & $\begin{array}{l}\text { Healed } \\
\text { fusion }\end{array}$ \\
\hline 6 & $\mathrm{P}$ & M & 28 & $\begin{array}{l}\text { Progressive } \\
\text { kyphosis C2- } \\
\text { C7 }\end{array}$ & 100 & 0 & $\mathrm{Y}$ & None & $\begin{array}{l}\text { Healed } \\
\text { fusion }\end{array}$ \\
\hline
\end{tabular}

$\mathrm{A} / \mathrm{P}=$ Anterior/Posterior, $\mathrm{P}=$ Posterior, $\mathrm{M}=$ Male, $\mathrm{F}=\mathrm{Female}, \mathrm{Y}=\mathrm{Yes}, \mathrm{N}=\mathrm{No}, \mathrm{mL}=$ milliliters, mo $=$ months 
Table 2: Comparison between Pre-op and Post-op Cobb angle and T1 Slope

\begin{tabular}{|l|l|l|l|l|}
\hline Subject ID & $\begin{array}{l}\text { Pre-op C2- } \\
\text { C7 Cobb } \\
\text { (degrees) }\end{array}$ & $\begin{array}{l}\text { Post-op C2-C7 } \\
\text { Cobb } \\
\text { (degrees) }\end{array}$ & $\begin{array}{l}\text { Pre-op T1 } \\
\text { Slope } \\
\text { (degrees) }\end{array}$ & $\begin{array}{l}\text { Post-op } \\
\text { T1 Slope } \\
\text { (degrees) }\end{array}$ \\
\hline 1 & 71 & 1 & 12 & 8 \\
\hline 2 & 111 & 11 & -43 & 18 \\
\hline 3 & 88 & 26 & 14 & 24 \\
\hline 4 & 91 & 43 & -21 & 18 \\
\hline 5 & 66 & -30 & -14 & 31 \\
\hline 6 & 74 & -16 & 30 & 24 \\
\hline
\end{tabular}

A negative value indicates lordosis 


\section{Pediatric Pathology All Grown Up - An Interesting Case of Adult Tethered Spinal Cord}

Dimitri Laurent, $\mathrm{MD},{ }^{1}$ Olgert Bardhi, $\mathrm{MS},{ }^{1}$ Jason Gregory, $\mathrm{MD},{ }^{2}$ Anthony Yachnis, MD, ${ }^{2}$ Lance S. Governale, MD ${ }^{1}$

Cervical myelopathy in an adult is typically the result of degenerative disease, trauma, or infection. Dysraphism is rarely the cause. The authors report the case of a 35-year-old male drywall installer who presented with two years of progressive left upper extremity weakness, numbness, and hand clumsiness. Only upon detailed questioning did he mention that he had neck surgery just after birth, however he did not know what was done. He then also reported that he routinely shaved a patch of lower back hair, but denied bowel, bladder, or lower extremity dysfunction. Magnetic resonance imaging of the cervical spine demonstrated T2 hyperintensity at C4-C5 with dorsal projection of the neural elements into the subcutaneous tissues concerning for a retethered cervical myelomeningocele. Lumbar imaging revealed a diastematomyelia at L4. He underwent surgical intervention for detethering and repair of the cervical myelomeningocele. Four months postoperatively, he had almost complete resolution of symptoms, and imaging showed a satisfactory detethering. The diastematomyelia remained asymptomatic and is being observed.

Affiliations:

${ }^{1}$ Department of Neurosurgery, University of Florida, Gainesville, Florida

${ }^{2}$ Department of Pathology, University of Florida, Gainesville, Florida

Corresponding Author:

Lance S. Governale, MD, FAANS, FAAP

Chief of Pediatric Neurosurgery

L.D. Hupp Associate Professor of Neurosurgery

University of Florida

McKnight Brain Institute, Room L2-100

1149 South Newell Drive

Gainesville, Florida 32611

Phone: (352) 273-9000

Fax: (352) 392-8413

Email: lance.governale@neurosurgery.ufl.edu

Disclosures for all authors:

None 


\title{
A Complex Case of Late Diagnosis of a Sagittal Synostosis with Intracranial Pressure
}

\author{
Eylem Ocal, MD \\ Department of Neurosurgery, University of Arkansas For Medical Sciences/ Division of Pediatric Neurosurgery, \\ Arkansas Children's Hospital, Little Rock, Arkansas, USA
}

A 5-year old boy presented with a few years history of progressive headaches and emesis. Recently, he was complaining of blurry vision. She was neurologically intact with the exception of papilledema. Imaging of the brain revealed sagittal synostosis, imprinting of the skull. Images performed at earlier ages for various reasons were normal.

Due to these findings with high intracranial pressure, surgery for cranial vault remodeling and sagittal synostosis repair was recommended. Surgery was complicated with significant blood loss starting from scalp incision. Patient required resuscitation during surgery. A strip craniectomy and barrel staves were completed safely and patient was transferred to intensive care unit post-operatively. He was discharged in stable condition but developed low pressure headache 6 weeks post-operatively. Papiledema resolved but patient was noted to have cerebellar sagging, clinical and imaging findings of low intracranial pressure. This was refractory to conservative measurement and required re- do Cranioplasty. This surgery was uneventful. Patient was discharged home with no significant headaches. However, he then developed recurrent headaches. An ICP monitor was placed for further diagnosis which showed low pressure. Patient remained neurologically stable and responded conservative headache treatment.

With this complex case, treatment options for delayed occurrence of craniosynostosis, related high intracranial pressure, rare occurrence of low intracranial pressure post- surgery and its treatment are discussed. 


\title{
Neuroendoscopic Evacuation of a Post-operative Intraventricular Empyema;
}

\section{Technical Pearl and Lessons Learned from this Post-operative Complication}

\author{
Violette M. Recinos, MD; Pablo F. Recinos, MD; and Kaine Onwuzulike, MD, PhD
}

Pyogenic ventriculitis is a rare but severe post-neurosurgical complication. The infection is often resistant to antibiotic treatment alone. Continuous intraventricular irrigation and drainage ("feed me, drain me" system) has been suggested but the technique can be cumbersome, increases the risk for secondary infection, and is often inadequate in removing adherent purulence. We hypothesize that early, aggressive evacuation of the purulence via neuroendoscopic approach and aspirator may improve outcomes and decrease long -term sequelae.

In this report, we review the case of a 17 year- old with a history of sub-total resection of a $4^{\text {th }}$ ventricular ependymoma and ventriculoperitoneal shunt (VPS) placement at an outside institution. Due to tumor and symptom progression, he underwent a re-do suboccipital craniotomy for gross total resection and conversion of his pre-existing VPS to an external ventricular drain (EVD) for post-operative monitoring and attempt at weaning for shunt independence. He failed the EVD wean and a subsequent attempt at an endoscopic third ventriculostomy, and therefore his VP shunt was replaced on POD\#11.

His post-operative course was complicated by gram-negative meningitis and wound infection 6 days later. He underwent wound washout, removal of the shunt and replacement with EVD. Over the next 5 days, despite aggressive EVD drainage and intraventricular and IV antibiotics, the patient remained febrile with worsening mental status. He developed thick purulence draining from the EVD. He was brought back to the OR for a right frontal endoscopic ventricular washout and was found to have purulence completely casting the ventricular system. A large portion of the pus was evacuated using irrigation and a side-cutting aspiration device. Post-operatively the patient clinically improved and his CSF eventually cleared. He required several more endoscopic procedures including septum pellucidotomy and aqueductal stenting to treat his post-infectious loculated ventricular system, and ultimately required the placement of bilateral VP shunt catheters. He had a prolonged recovery period but eventually recovered enough to be discharged from the rehabilitation center.

Five months later, he developed worsening upper extremity weakness and was found to have a holocord syrinx secondary to adhesions at the level of the foramen magnum. This required a re-do suboccipital surgery for dissection of adhesions and placement of a $4^{\text {th }}$ ventricular stent. He improved post-operatively and eventually returned to his home country of origin. Unfortunately, he died there 2 years later secondary to complications of shunt malfunction.

In the 2 cases of post-operative ventriculitis that we have encountered since this complication, we have performed early (with 1-2 days) endoscopic intraventricular washout of the purulent CSF with the side-cutting aspirator and have seen good clinical results. This appears to have resulted in earlier CSF sterilization and decrease in the occurrence of post-infectious, loculated ventricles and scarring. 


\title{
Early Endoscopic Ventricular Irrigation of Gram Negative Meningitis Prevents Loculated Hydrocephalus
}

\author{
Michael Burke, MD
}

Every member of this audience is aware of the complications associated with gram-negative meningitis and ventriculitus in premature infants and neonates. The standard of care is the neonatologist will start them on antibiotics and then pediatric neurosurgeons will be consulted to deal with the hydrocephalus that evolves into multiloculated ventricles. Multiple surgical procedures and or shunts are performed on an intellectually devastated child only to be discharged with ongoing follow-up and revisions of shunts. Endoscopic fenestration of the loculated ventricles can cut our losses with respect to the number of catheters and shunts required. Frustrated with this outcome and clinical challenge the author requested the neonatologist notify me of this diagnosis immediately. The patients are taken to surgery where an endoscopic ventricular irrigation and drainage utilizing lactated ringer's and Gentamycin is performed until all flocculence and the CSF is cleared. An EVD is left in place to sample CSF and tap to control hydrocephalus. While the intellectual outcome is unchanged and the children still require treatment for hydrocephalus, this proactive approach has led to complete resolution of loculated hydrocephalus. A brief surgical video will be presented. 


\title{
Case Presentation: Acquired Chiari Malformation Secondary to Thoracic Cyst and Tethered Spinal Cord
}

\author{
Holly Gilmer, MD
}

This right-handed female presented to clinic at 8 years of age. She was the product of a FTSVD with left shoulder dystocia. She had a mild hemiparesis that resolved with PT by 14 months. She did not have a brachial plexus injury. She walked at 10 months, and met all of her developmental milestones. She has had a left ptosis since birth, and didn't open her left eye for 2 weeks. She had had 3 left eye surgeries for this, most recently 2013. She had speech delay, and didn't talk until she was 3 . She had frequent headaches, neck pain, back pain, and BLE pain. She has numbness and tingling in her feet. She frequently complained of "jumpy legs", which sometimes kept her up at night. She began dribbling urine about 4 years of age, after being fully potty-trained. At presentation, she was still dribbling urine, and wearing a pad. Her past medical history was also significant for ASD, ADHD, and hereditary spherocytosis. On examination, she had a left ptosis, a diminished gag reflex and bilateral dysmetria.

Cervical spine MRI showed a Chiari I malformation, with tonsillar herniation down to the middle third of C1, and a lobulated epidural arachnoid cyst at T4-T7(Figure 1 ). Of note, there was no MRI evidence of spinal cord tethering at the level of the cyst. Lumbar spine MRI showed a tethered spinal cord, with a low-lying conus down to L3, a filar cyst, and a thickened, dorsal filum (Figure 2).

On 2/19/18, she underwent L2-3 laminectomy, spinal cord untethering, and T4-7 laminectomies with ligation of the arachnoid cyst and repair of CSF leak. Profound tethering of her thoracic spinal cord was found to the cyst, as well as a large dural defect with CSF leak (Figures 3 and 4). The cyst was ligated and the spinal cord untethered at this site (Figure 5). At L2-3, a thickened filum was found tethered dorsally to the dura (Figure 6).

At her 6-week postoperative visit, her mother reported no further urinary dribbling which had gone on since 4 years of age. She was no longer in pull-ups. Her dysmetria was gone, and her left ptosis was improved. At 7 months post-op, she was still continent, with no headaches, neck pain, back pain, or leg pain. Her numbness/tingling in her extremities and her ptosis had resolved.

Postoperative brain and spine MRI showed no signs of tethering at the thoracic or lumbar site. The conus had ascended from L2-3 to L1. The thoracic cyst was decompressed (Figures 7 and 8). The Chiari malformation has ascended from middle $\mathrm{C} 1$ to above the $\mathrm{C} 1$ ring, with expansion of the brainstem and increased CSF flow around the cerebellar tonsils (Figure 9).

This patient presented with symptoms and signs of tethered spinal cord and Chiari malformation. A thoracic arachnoid cyst with spinal cord tethering at T4 and L2 was responsible for her cerebellar tonsillar herniation and symptoms. In this case, the typical initial treatment of epidural blood patch would not have been effective, but her symptoms resolved completely with ligation of the cyst and spinal cord untethering. 


\section{Figure 1}
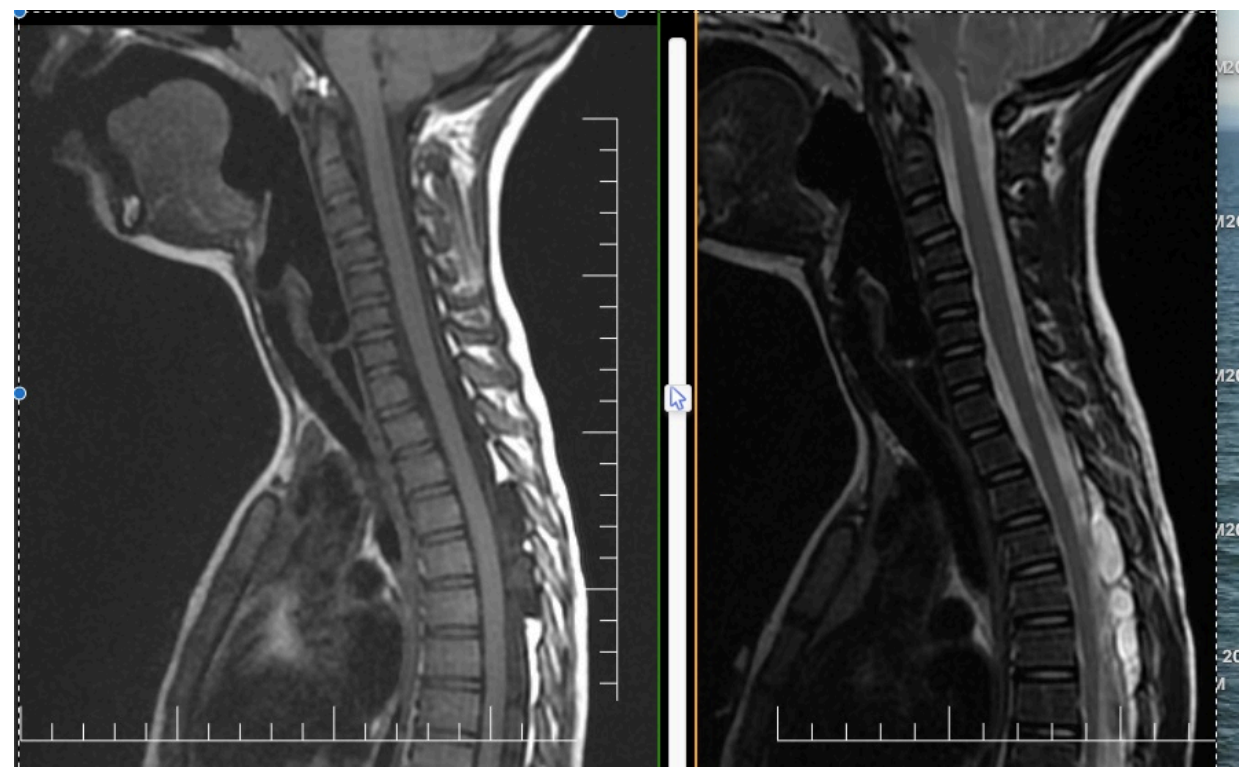

Figure 2
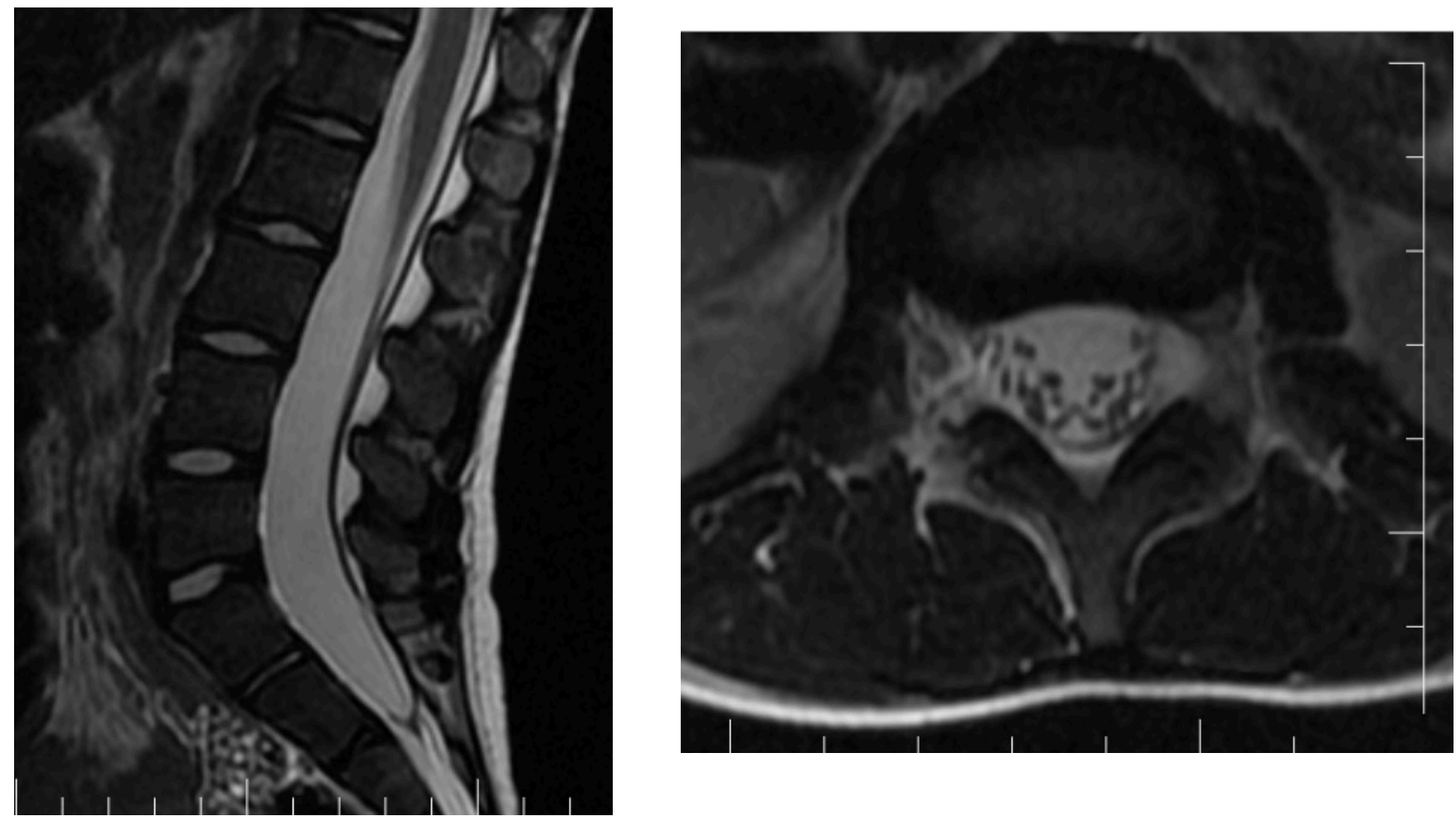
Figure $3-4$

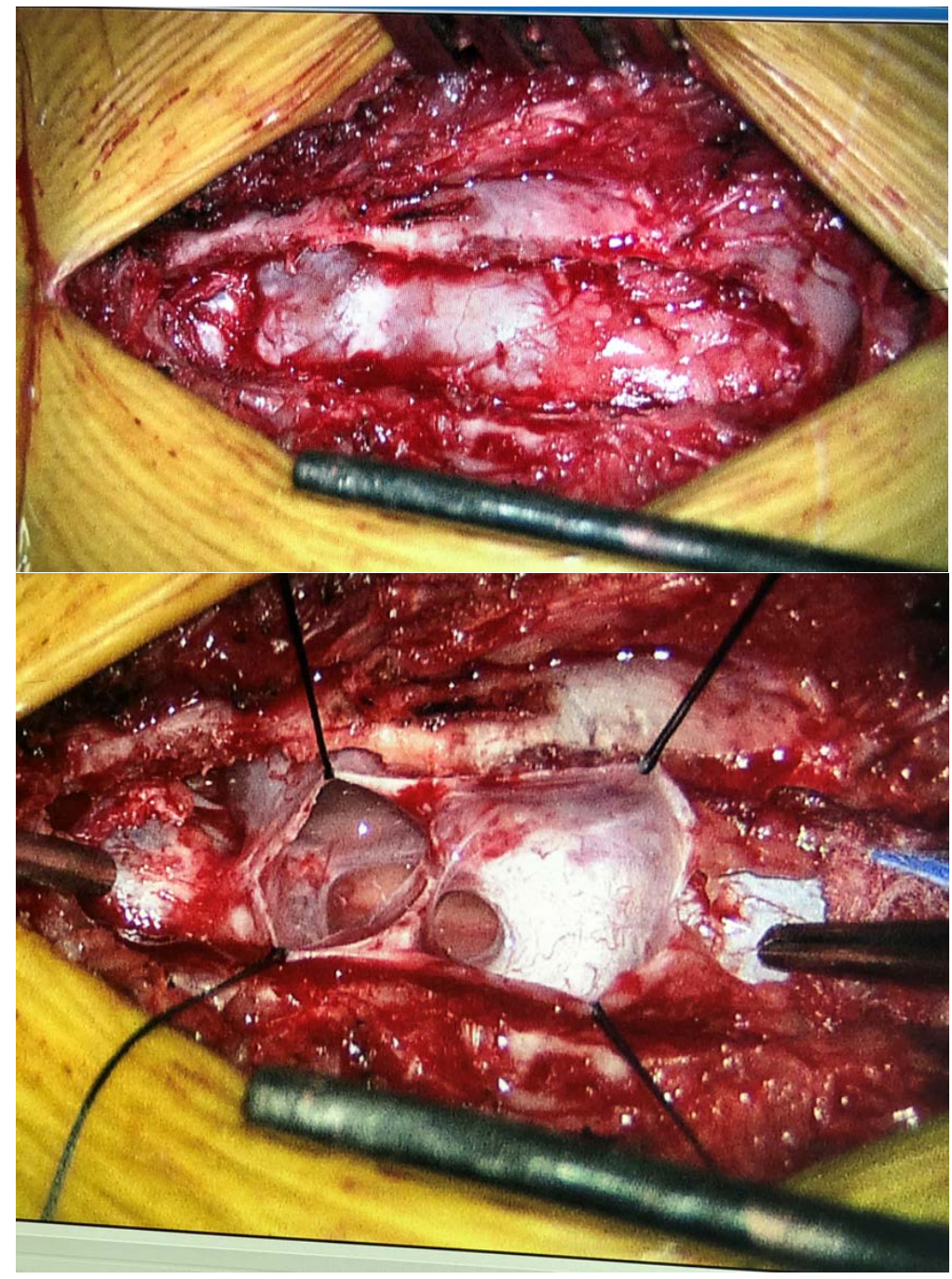

Figure 5

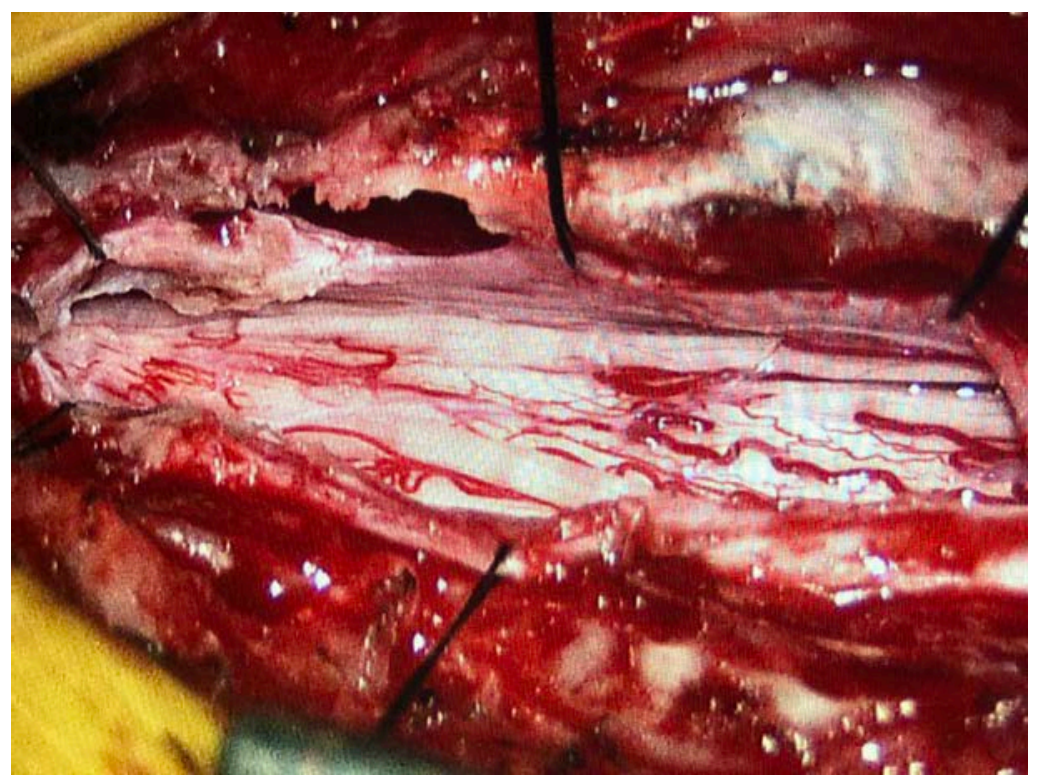


Figure 6

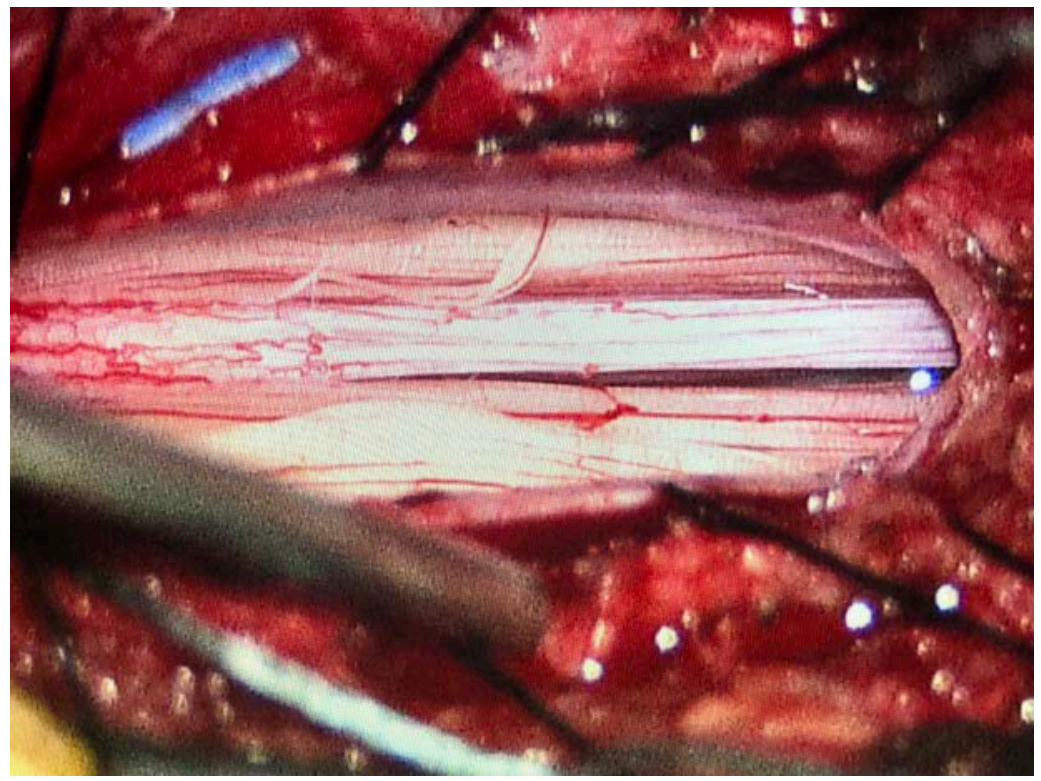

Figure 7-8
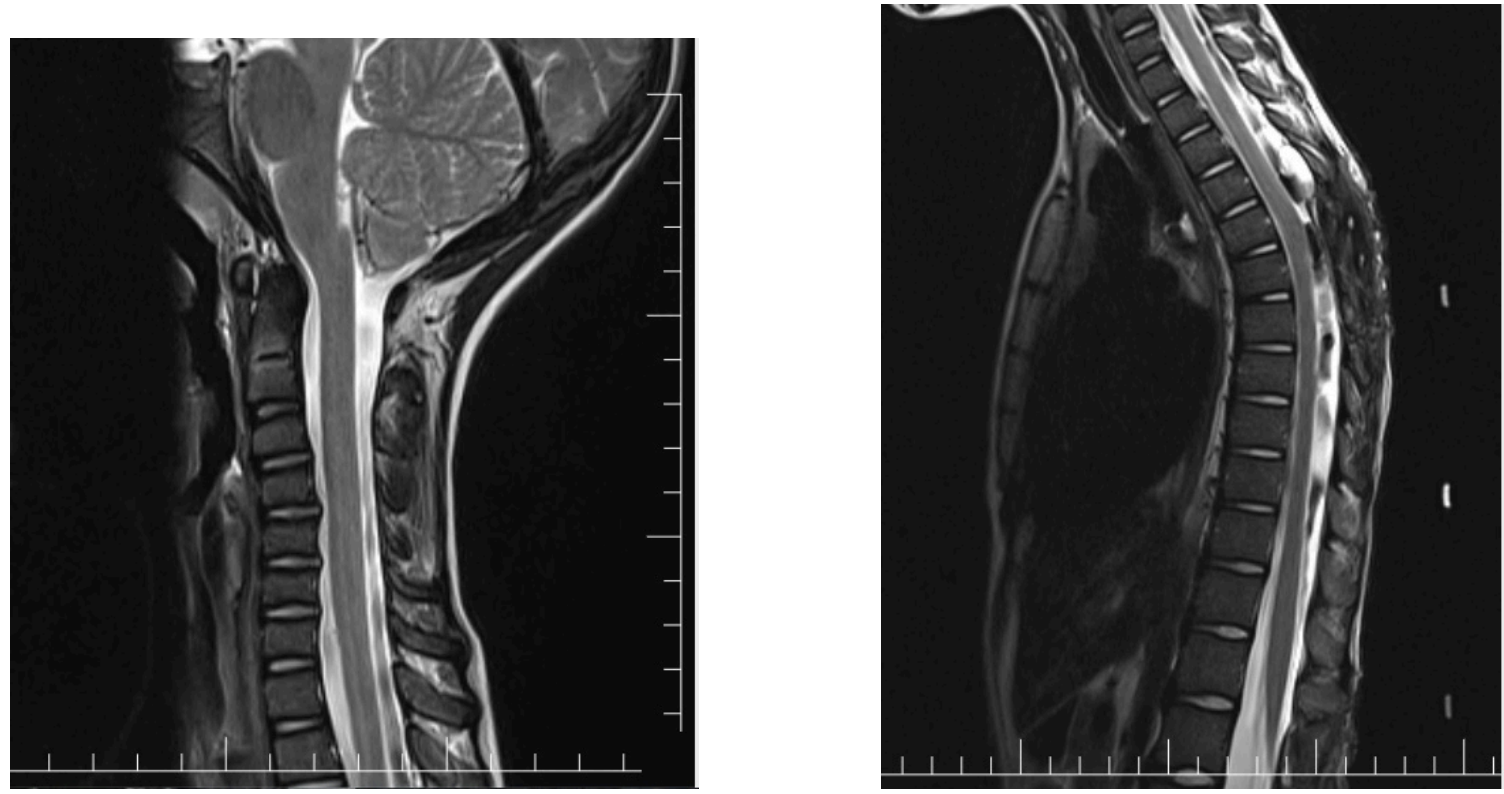
Figure 9

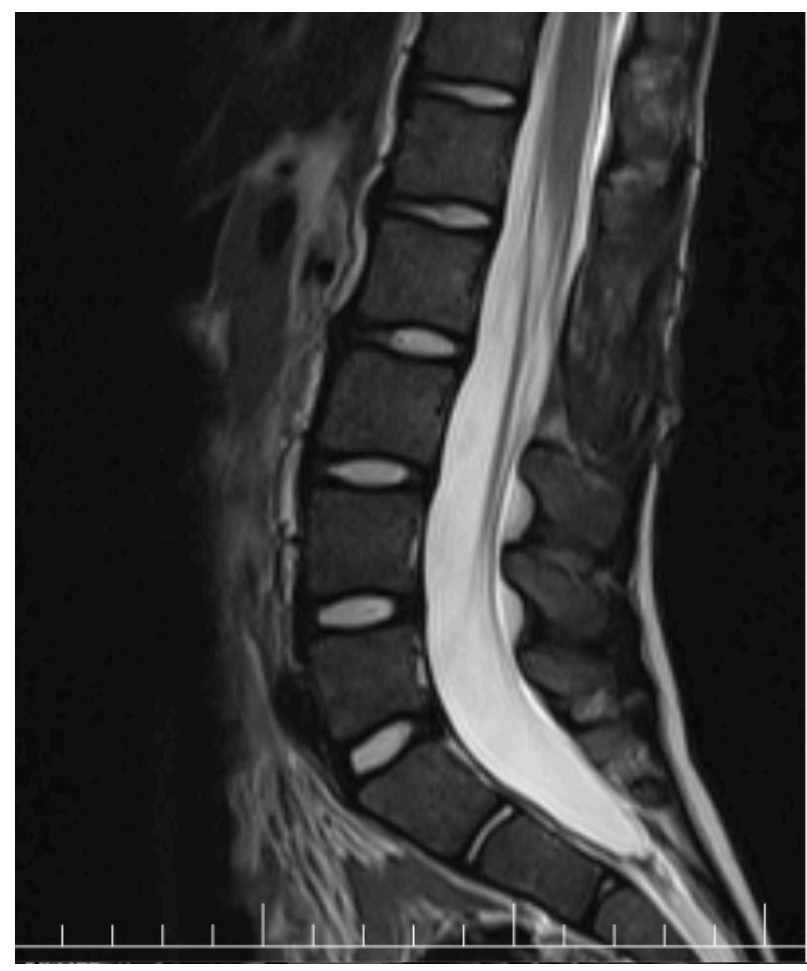




\title{
Subpial Tonsil Resection for Immediate Disimpaction of the Cervicomedullary Junction
}

\author{
Paul Klimo
}

\section{Intro}

Decompression of the cervicomedullary junction (CMJ) and restoration of CSF circulation at the outflow of the fourth ventricle and foramen magnum are critical objectives in the surgical management of $\mathrm{CM} 1$ and 1.5 malformations. This is accomplished through a variety of techniques but most commonly through sub-occipital craniectomy with duraplasty. If the dura is opened, further decompression can be achieved through tonsillar fulguration, but some have argued against the use of this technique. Here we present our experience of subpial tonsillar resection as a means to immediately and aggressively decompress the cervicomedullary junction and reestablish unimpeded CSF flow from the 4th ventricle.

\section{Methods and Surgical Technique}

We retrospectively collected and reviewed data on a single surgeons series of sub occipital craniectomies with subpial cerebellar tonsil resection for the treatment of primarily Chiari malformations. Children with posterior fossa tumors with severe impaction of the CMJ were also included. The technique is briefly described. After opening the dura, the "waist" of the pyramidal shaped cerebellar tonsil is cauterized in a transverse fashion and then opened sharply. A subpial resection is then carried, until the obex of the 4th ventricle (medially) and the cerebellomedullary angle (lateral) is fully visualized. Once both tonsils are resected, the inferior pial edge of the resection cavity is sutured up to the cerebellar surface so as to bring the pia "up and out", thus fully exposing the outflow of the 4th ventricle and each respective cerebellomedullary angle. Dural patch graft is then placed followed by standard wound closure.

\section{Results}

Pending

\section{Conclusion}

Subpial tonsillar resection is a safe and highly effective means to immediately and definitely decompress the CMJ. It has a number of key advantages over duraplasty alone or duraplasty with fulguration. 


\title{
Cannabinoids and Cancer - Case Report
}

\author{
Monica C. Wehby, MD, FAANS, FACS
}

Cannabinoids in the form of both Delta 9 tetrahydrocannabinol (THC) and cannabidiol (CBD) are known to be effective in treating pain, nausea, and loss of appetite in cancer patients. In addition, evidence also is emerging that some cannabinoids can inhibit angiogenesis, impede growth of cancer cells, and induce apoptosis and autophagy (Kenyon 2018).

In this case report, a 19-year-old Russian female underwent partial resection of a butterfly glioblastoma 5 years prior, and the family elected not to pursue further treatment due to her poor prognosis. Other than mild facial weakness and seizures, she did well with very slow progression of several residual enhancing nodules within the tumor cavity. Four years later, there was significant progression of the enhancing nodules, prompting a stereotactic excisional biopsy which the pathologist read as slightly lower grade than the original biopsy. Subsequently, four months post-op, a residual enhancing nodule increased in size and new nodules appeared. They remained stable on follow-up scan one month later. The family again opted out of adjuvant therapy.

Six months later, follow-up scan showed disappearance of two enhancing nodules with overall decrease in enhancing disease. Upon questioning, mother admitted that for the past 4 months, she had been administering highly concentrated CBD oil that she had obtained from Europe. The patient had received no other treatment than her anticonvulsants.

Although studies are in their infancy, Cannabinoids could possibly be useful in treating brain tumors and other forms of cancer.

No disclosures 


\section{Impact of Infantile Epilepsy Surgery on Neurodevelopment in Tuberous Sclerosis Complex}

Jeffrey P. Blount MD, Aria Fallah MD MS, Joseph R Madsen MD, Scellig S Stone MD PhD, Howard L. Weiner MD, Leslie Grayson, MD, Jurriaan M Peters, MD, PhD, Tarrant McPherson, MA, Darcy A Krueger, MD, PhD, Mustafa Sahin, MD, PhD, Joyce Y Wu, MD, Hope A Northrup, MD, Brenda Porter, MD, Gary R Cutter, PhD, Sarah E O'Kelley, PhD, Jessica Krefting, RN and E. Martina Bebin, MD, MPA, on behalf of the TACERN Study Group.

\section{Introduction}

Children with Tuberous Sclerosis (TS) are at elevated risk for pronounced neurocognitive and behavioral impairment. More than $60 \%$ experience seizure onset in the first year of life during a critical period of neurodevelopment, with medical resistance in up to two-thirds of cases. It is unknown whether improved seizure control imparted by successful epilepsy surgery can mitigate these effects. We investigated whether aggressive surgery in infancy ( $<2$ years age at time of surgery) favorably impacts neurodevelopmental outcomes in a multi-center cohort of young children with TS. Our hypothesis was that young children with refractory epilepsy would experience an improvement in neurodevelopmental outcome if the clinically detrimental, targeted seizure type was eliminated surgically - whether or not total seizure freedom was attained

\section{Methods}

We identified and studied infantile (median age 17 months, range 3.7-21.3 months) surgical cases $(n=19)$ from a prospective, multicenter observational study of 160 children with TSC. Single stage open resections guided by intraoperative electrocorticography were most common, followed by procedures with intracranial electrodes. Seizure outcomes were characterized relative to the specific seizure type targeted by the surgery. Neurodevelopment assessment was performed by obtaining serial Mullen Scales of Early Learning (MSEL), Vineland Adaptive Behavior Scales (Vineland-II) and Preschool Language Scales (PLS-5) at 3, 6, 9, 12, 18, 24 and 36 months of age. Seizure outcomes, complications and neurodevelopmental metrics were compared to children without seizures, with controlled seizures and with refractory seizures.

\section{Results}

Seizure outcomes were excellent in 10 (53\%, Engel I), favorable in 12 (63\%, Engel I-II), and poor in 7 (37\%, Engel III-IV), 9 (47\%) had new or ongoing seizures distinct from the targeted type. Over time, all children with seizures demonstrated decline or attenuated gains in MSEL, Vineland-II, and PLS-5. The surgical group scored lowest, followed by the refractory, medically controlled, and without-seizure groups. Favorable surgical outcome was associated with an increase of PLS-5 mean standard scores, and expressive language sub-scores of MSEL and PLS-5, at times surpassing the refractory seizure group.

\section{Conclusions}

In TSC, early surgical intervention is associated with neurodevelopmental gains. This implies some potential for epilepsy surgery to favorably impact the natural history of neurocognition and behavior. 


\title{
Granger Causality in Stereoelectroencephalography: Interictal Data Predict Ultimate Seizure Onset Zone and Resection
}

\author{
Joseph R. Madsen ${ }^{1}$, Scellig Stone ${ }^{1}$, Eun-Hyoung Park ${ }^{1}$, Phillip L. Pearl ${ }^{2}$ \\ Departments of Neurosurgery ${ }^{1}$ and Neurology ${ }^{2}$, Boston Children's Hospital, Harvard Medical School, \\ 300 Longwood Avenue, Boston, Massachusetts 02115, USA
}

\section{Disclosures}

The authors have no conflicts of interest or financial ties to disclose.

\section{Background}

Stereoelectroencephalography (sEEG) is increasingly utilized for invasive localization of ictal onsets in patients with medically refractory focal epilepsy. Recently we have published that Granger Causality (GC) analysis can statistically predict seizure onset and resection zones using interictal intracranial EEG (iEEG) data obtained from subdural grids alone or together with conventional depth electrodes. In this study, we investigated whether GC technique applied to interictal sEEG data can predict ictal onset and resection zones.

\section{Objective}

To examine whether interictal SEEG data can produce GC maps that statistically resemble the topography of the ictal onset zone and resection zone.

\section{Methods}

Following sEEG electrode implantation, the first 10min of data collected after all channels were connected was analyzed $(n=20)$. Causality maps were quantitatively compared to conventionally-constructed surgical plans and resections, by using rank-order statistics.

\section{Results}

In 19 of 20 cases, the interictal GC rankings of electrodes mapped to the seizure onset and early spread zones had higher causality than predicted by chance (range: $p<10^{-5}$ to 0.03 ). In 17 of 18 cases that went on to resection or ablation, causality in the resection zone was significantly increased (range: $p<10^{-5}$ to 0.03 ). The aggregate probability of such a match is very small $\left(p<10^{-38}\right)$, suggesting that the networks highlighted in interictal GC maps correlate with surgically-relevant seizure networks.

\section{Conclusion}

GC analysis applied to sEEG data has the potential to help identify ictal onset and predict resection zones from interictal data. The statistical link between high interictal GC loci and ultimate seizure onset and resection zones appears even stronger with sEEG than with subdural recordings, and it is feasible that intraoperative GC calculations could help guide selection of targets for sEEG electrode placement. 


\title{
Two-level Laminectomy for Conus Medullaris-level Combined Dorsal and Ventral Lumbar Selective Rhizotomy for Treatment of Mixed Spasticity and Dystonia: Technical Note and Complications
}

\begin{abstract}
Robert P Naftel, MD
Combined dorsal and ventral lumbar rhizotomy has been described for treatment of mixed spasticity and dystonia. Although conus medullaris-level selective dorsal rhizotomies are commonly performed and reported, combined dorsal and ventral rhizotomies have only been reported using an L1 to S1 laminectomy/plasty. Four children failing other treatments of their mixed dystonia and spasticity underwent a two-level laminectomy for conus-level combined selective rhizotomy. A technique will be described to perform a conus medullaris-level combined dorsal and ventral rhizotomy through a 2-level laminectomy. In these 4 patients (median age 14yo), all underwent palliative treatment of their mixed tone. Three patients had failed baclofen pump management and one patient was not a candidate for baclofen pump therapy due to lack of hospital access. All patients had scoliosis and three had been fused. Dorsal and ventral roots from L1 to S2 were isolated, divided into rootlets, stimulated with neurophysiology, and cut $60-69 \%$ of dorsal rootlets and $60-69 \%$ of ventral rootlets. Two patients required in-and-out catheterization ranging from 1 to 5 days with bladder function returning to baseline thereafter. No patient experienced infection, CSF leak, or other complication. Average length of stay was 3 days. Postoperative tone response pending longer follow up for formal measurement at 1 year.
\end{abstract}




\title{
Disconnection Surgery for Frontal Lobe Epilepsy
}

\author{
James Baumgartner MD, Angel Cordero MD, Amy Waller PA-C, \\ Ki Hyeong Lee MD, Joo Hee Seo MD
}

\begin{abstract}
The frontal lobe (FL) is often an integral part of the epilepsy network in medically refractory epilepsy (MRE). Especially for non-lesional cases, FL resection has yielded disappointing results. $\mathrm{FL}$ disconnection may offer an alternative to $\mathrm{FL}$ resection.

Methods

We performed a retrospective review of patients treated with FL disconnection as part of the surgical management of their MRE. All disconnections were preceded by intracranial video-EEG monitoring. After 2016 stereo-EEG electrode monitoring was also performed. Patient demographics, clinical presentation, procedural complications and clinical and imaging followup were assessed. The disconnection procedure included division of the anterior cingulum.

\section{Results}

Twenty-two patients were identified who underwent FL disconnection between March 2011 and November 2017 at our institution. There were ten male and twelve female patients with a mean of 11 years of age (range 6 months to 47 years). Surgical complications included 1 epidural hematoma, 1 wound infection and one transient case of SIADH. Four patients experienced transient disconnection type symptoms, but no true SMA syndrome cases occurred. Of 13 cases who underwent right sided FL disconnection surgery, 5 achieved ILAE class 1 outcome and 8 experienced no improvement in seizure control at last follow-up. Of 9 cases who underwent left sided disconnection surgery, 7 achieved ILAE class 1 outcome, 1 achieved ILAE class 2 outcome and 1 remained at ILAE class 5 . Outcomes were superior for younger patients than for older patients (ILAE Class 1 or 2 for eight of eleven patients less than 21 years of age and for five of eleven patients more than 21 years of age).
\end{abstract}

\section{Conclusion}

This small series suggests that FL disconnection is well tolerated and may have a role in the management of complex MRE. 


\title{
Is Asleep Pediatric Deep Brain Stimulator Electrode Placement the New Gold Standard?
}

\author{
John Honeycutt, Stephanie Acord, Fernando Acosta, and Warren Marks
}

\section{Introduction}

Pediatric deep brain stimulator (DBS) surgery is becoming a more common surgical treatment option to addresspediatric movement disorders. Early in our experience, patients underwent microelectrode recording (MER) and intraoperative testing while awake to assure correct electrode placement. In 2013, we began to employ intraoperative MRI (iMRI) to assist targeting and confirmation of correct lead placement in anesthetized patients, reasoning that "asleep surgery" would reduce operative time while increasing patient comfort without jeopardizing outcome.

\section{Methods}

A retrospective review of 110 pediatric patients undergoing DBS electrode placement at Cook Children's Hospital from 2013 through 2017compared length of surgery, complication rate, and outcomes for the two groups.

\section{Results}

110 patients underwent 130 procedures for electrode placement (20 electrode revisions). Ninety-six awake patients underwent DBS electrode placement surgery assisted by MER. Thirty-four patients underwent DBS surgery guided by iMRI while under general anesthesia. The groups possessed similar demographics. Duration of of "asleep surgery" procedures (3.5 hours) was significantly shorter ( $p>.001$ ) than that of awake surgery (5.4 hours). No patient undergoing asleep surgery required lead revision, compared to 20 patients in the awake surgery group ( $p>.003)$. The complication rate for asleep surgery was $3 \%$ and $31 \%$ for asleep and awake groups, respectively $(p>.004)$. The outcome assessments for the two groups was identical, except for the one- year BFM Disability scale, which disclosed significant improvement for the asleep series patients compared to those in the awake series.

\section{Conclusion}

Asleep pediatric DBS electrode placement using intraoperative MRI guidance has significantly decreased operative time, complication rate, and incidence of electrode revision without altering functional outcome. In fact, need for electrode revision has disappeared following adoption of asleep surgery for DBS placement. DBS electrode placement under general anesthesia has become the "gold standard" at our institution. 


\title{
Epilepsy Surgery in Young Children with Tuberous Sclerosis Complex: A Novel Multi-Modal Surgical Approach
}

\author{
Vijay M. Ravindra, M.D., MSPH*1,2, Tyler Lazaro, M.D.*2, Patrick Karas, M.D. ${ }^{2}$, Al-Wala \\ Awad, M.D. ${ }^{4}$, Ilana Patino, PA-C ${ }^{1}$, Emily E. Edens, PA-C ${ }^{1}$, Rohini Coorg, M.D. ${ }^{3}$, Irfan Ali, \\ M.D. ${ }^{3}$, Daniel J. Curry, M.D. ${ }^{1,2}$, Howard L. Weiner, M.D. ${ }^{1,2}$
}

\section{Background}

Tuberous sclerosis complex (TSC) is associated with medically refractory epilepsy and developmental delay in children; seizure onset is early in life, thus functional and developmental outcomes may improve with seizure control. Tailoring treatment is essential for children with TSC - thus we have adopted a multi-modal surgical approach consisting of invasive intracranial monitoring and surgical treatment.

\section{Objective}

To describe a multi-modal surgical diagnostic and therapeutic approach to epilepsy associated with TSC and compare outcomes and re-treatment for seizure focus resection (SFR) versus laser interstitial thermal therapy (LITT).

\section{Methods}

Clinical and radiographic data were collected prospectively collected on a consecutive cohort of children treated at a single, tertiary academic institution with a multidisciplinary approach to epilepsy. Treatment strategies were compared for seizure freedom, developmental improvement, Engel class. Kaplan Meier survival analysis was performed to evaluate time to re-treatment.

\section{Results}

Thirty-eight children (20 females, 18 males) with TSC underwent surgical epilepsy treatment by two surgeons from May 2016 - April 2019 - 37 underwent stage II invasive monitoring - 21 SEEG (18 underwent LITT, 3 SFR, $p<0.05$ ) , 16 craniotomy for grids/electrodes (all underwent $S F R, p<0.05$ ). The mean age differed between the two (SFR 2.5 years vs. LITT 5.4 years, $p=0.002$ ). Patients undergoing SFR suffered more seizures (49 vs. 9, $p=0.016$ ) and were on more AEDs (3.6 vs. 2.6, $p=0.024$ ) preoperatively. 19 underwent SFR and 19 underwent LITT as primary treatment. The median Engel class at last follow up was 2 for both groups, and there were similar rates of children with either Engel class 1 or 2 in the LITT group (66\%) vs. SFR (53\%) ( $p=0.407)$. The mean follow-up time was longer in the SFR cohort (1.96 years vs. 1.3 years, $\mathrm{p}=0.065)$. Children treated with LITT were more likely to have achieved a $50 \%$ reduction in seizures after initial surgical treatment $(84 \%$ vs. $63 \%, p<0.05)$ with similar rates of developmental improvement ( $53 \%$ vs. $79 \%, p=0.24)$. Sixteen patients required secondary surgical treatment and two required a third. Three patients initially treated with LITT subsequently went on to undergo surgical resection as a secondary treatment. Seven patients initially underwent resection then underwent LITT. The mean time to secondary surgery was 297.8 days vs. LITT 577 days ( $p=0.09$ ). 


\section{Conclusions}

A multi-modal surgical approach can be useful in treating children with TSC associated epilepsy. We have successfully reported the use of open and minimally invasive diagnostic and therapeutic techniques. Long-term follow up and randomized trials focused on treatment method are needed to adequately compare the treatment modalities, but they can be used effectively in a complimentary fashion.

\section{Key Words}

Tuberous sclerosis; stereo-electroencepahalography, craniotomy

Running Title: Multi-modal surgical treatment for TS

\section{Disclosure}

The views expressed herein are those of the author(s) and do not necessarily reflect the official policy or position of the Department of the Navy, Department of Defense, or the U.S. Government. 


\title{
Early Resection of MRI Abnormalities in Patients with Li Fraumeni Syndrome
}

\author{
Myseros JS, Keating RF, Oluigbo CO, Bornhorst M
}

\section{Introduction}

Li Fraumeni Syndrome (LFS) is an inherited familial predisposition to tumors. Surveillance imaging may reveal cortical brain lesions, rarely symptomatic, concerning for gliomas. Given their diffuse, innocuous appearance, they are oftentimes followed with serial imaging. We present a cohort of patients presenting with such lesions and their management.

\section{Methods}

Five patients with LFS and screening brain MRI were found to have diffuse T2 cortical abnormalities. Three were female and two male, one female and one male being siblings. The ages were 6-20 years. With the exception of the male sibling, the other four patients underwent resection of their lesions upon initial imaging. The male sibling was followed with serial imaging, and upon progression of the lesion, underwent resection.

\section{Results}

All patients tolerated the procedures well. With the exception of the male sibling, all four patients had histology consistent with diffuse low grade glioma. The male sibling was diagnosed with High Grade Neuroepithelial Tumor. Three of the four diffuse tumors were positive for IDH1 mutation. The male sibling's tumor harbored a FLT1 and SMARCA4 mutation, and he has required additional therapy for diffuse disseminated disease within 12 months of initial surgery. The other four patients are well without recurrence at an average follow up of 24 months (1350 months).

\section{Discussion}

Patients with LFS have a predisposition for malignant transformation of innocuous appearing brain lesions found on screening MRI. In our cohort, we found that many of these tumors harbor IDH1 mutations, a mutation commonly found in secondary adult high grade gliomas. In the absence of the IDH1 mutation, other aggressive mutations can be seen. Given the propensity for and subsequent nefarious nature of these tumors, early surgery should be strongly considered as this allows for molecular stratification and may prevent the development and progression of a fatal tumor.

\section{Disclosures}

All four authors have no actual or potential conflict of interest in relation to this presentation. 


\section{Non-Resection Management of Craniopharyngiomas}

Paul Klimo

\section{Introduction}

Craniopharyngiomas are rare embryonic-based neoplasms of the sellar and parasellar region. They have a bimodal age distribution, and are often characterized by both solid and cystic components and calcifications. The tumor and its treatment can result in significant issues due to the proximity of critical neural, vascular, and endocrine structures. Surgical management continues to ignite debate with some advocating for aggressive resection whereas others favor a more limited resection followed by adjuvant therapy. However, a 3rd option exists that has not received as much attention - non-resection. This study focuses on a select group of patients whose tumor is either monocystic, or having a dominant cyst and were treated with cyst fenestration or catheter decompression followed by proton beam radiation.

\section{Results}

Thirty-three patients at our institution who underwent one of 3 techniques over the past 15 years: 1) frontal craniotomy for catheter placement into the cyst under direct visualization ("open" Ommaya); 2) stereotactic catheter placement into the cyst via a burr hole ("burr hole" Ommaya"); and 3) transsphenoidal fenestration of the cyst. The median age was 8.2 years with 21 (64\%) being male. Pure cystic tumors were present in 16 patients (48\%) whereas the other 17 patients had both solid and cystic. 31 out of $33(94 \%)$ had preoperative symptoms. The median tumor volume size was $5.2 \mathrm{~cm} 3$ (open Ommaya), $17.2 \mathrm{~cm} 3$ (burr hole Ommaya) and 4.7 (transsphenoidal).

\section{Conclusion}

Non-resection management of purely or predominantly cystic craniopharyngiomas should be considered first-line therapy in an effort to avoid well-known surgical morbidities. 


\title{
Utility of Lower Species Modeling of Human Brain Tumours: Identification of New Therapeutic Targets
}

\author{
Sick Kids Hospital, Division of Neurosurgery, \\ Department of Surgery, \\ University of Toronto
}

James Rutka, Uswa Shahzad, Michael Taccone, Brent Derry, and Joji Ishida

Email: james.rutka@sickkids.ca

Lower species model organisms such as Drosophila melanogaster, Caenorhabditis elegans (Celegans) and Danio rerio represent underappreciated alternatives to genetically engineered mice that fill this void. In recent years, a resurgence of interest in lower species modeling has led to the identification of new genes, targets, as well as technologies for high-throughput genetic and chemical manipulation. These lower species are advantageous in that their lifespans are short, they have high homology to the human genome, tumor latency is shorter, and numerous drug screens are possible. In C-elegans, we have created the let-60 (KRAS) and daf-18 (PTEN) mutant worm as a model of hyperproliferation and invasion. These two genetic lesions are frequently found in human high grade gliomas (HGGs). These worms develop the multivulvar (MUV) phenotype. Using the MUV worm, we have screened all 6 C-elegans chromosomes and over 20,000 genes to identify 14 novel genes that have not been described previously for HGGs which can be inhibited to reduce the proliferative and invasive phenotype of the MUV worm. Some of these novel hits include the genes for Huntingtin Interacting Protein 1 (HIP1), sphingomyelin phosphodiesterase 1, and Patched domain containing 1 (PDC1). Interestingly, these and other genes show relevance in terms or amplifications or deletions in the TCGA and other human cancer databases. Treatment of human HGG cell lines with inhibitors of these genes leads to decreased proliferation and invasion. This study demonstrates the potential value of screening lower species mutant organisms to rapidly determine novel targets for human diseases such as brain tumours. 


\author{
Netrin-1/Neogenin as DIPG Theranostic \\ Julie Sesen, Ph.D. ${ }^{1}$, Jessica Driscoll ${ }^{1}$, Edward Smith M.D. ${ }^{1,2}$ \\ ${ }^{1}$ Boston Children's Hospital, Vascular Biology Program \\ Boston Children's Hospital, Department of Neurosurgery
}

\title{
Introduction
}

Diffuse Intrinsic Pontine Glioma (DIPG) is a tumor with significant mortality, due largely to its capacity to invade adjacent normal brain. We have previously published studies identifying the axon guidance factor netrin-1 and its receptor neogenin as key drivers regulating brain tumor invasion in medulloblastoma and glioblastoma. Here we expand this work to investigate the role of netrin-1 and neogenin as putative DIPG theranostics; using them as paired biomarkers and druggable targets to reduce tumor invasion.

\section{Methods}

Urine of 49 DIPG patients and 42 age- and sex-matched controls was collected. Netrin-1 expression was quantified by ELISA. Results were subjected to univariate and multivariate statistical analyses and compared between clinical cohorts. In vitro experiments with established DIPG cell lines utilized RTqPCR, Western blot and flow cytometry to quantify expression of netrin1 and neogenin. The effect of neogenin blockade on viability and invasion was assessed by flow cytometry $(n=3)$ and transwell chamber assays $(n=3)$ respectively.

\section{Results}

Patient samples demonstrated significantly reduced urinary netrin-1 compared to matched controls $(p<0.005)$. This result was corroborated with cell lines in vitro. Neogenin was upregulated in these cells when compared to control glial cells when quantified by mRNA, protein and FACS analysis. Blockade of the neogenin target in DIPG cell lines resulted in a significant increase of cell death and a significant decrease of cell invasion.

\section{Conclusion}

Expanding on previous work, these data suggest an important role for netrin-1 / neogenin as regulators of tumor invasion and survival in DIPG. Urinary levels of netrin-1 may have utility as a theranostic, combining a non-invasive biomarker for DIPG and with a cell-surface druggable target, capable of reducing tumor invasion and increasing tumor cell death. 


\title{
Proteomic Analysis of Adamantinomatous Craniopharyngiomas Reveals Clustering with BRAF Low Grade Gliomas
}

\author{
Adam C. Resnick, Pichai Raman, Yuankun Zhu, Elizabeth M. Appert, Ximena Cuellar, Jena \\ V. Lilly, Pei Wang, Brian Rood, Phillip B. Storm; Shih-Shan Chen, MD and CPTAC \\ Collaborators
}

Genomic characterization has allowed for the differentiation of different tumor types based upon the abundance of gene transcripts. However, especially in childhood brain tumors, owing to the many layers of regulation between transcript and the posttranslationally modified protein, the functional moiety of the cell, it has been challenging to fully extrapolate biological signals from these transcriptional differences that could drive clinical translation. We hypothesized that a comparative analysis of the genome, proteome and phosphoproteome across 7 childhood brain tumors would yield a deeper understanding of the differences in their functional biology.

We performed tandem mass tag labeling and triple mass spectrometry of 219 fresh frozen tumor samples collected at CHOP as part of the Children's Brain Tumor Consortium, representing the histologic diagnoses of: high grade astrocytoma (26), low grade astrocytoma (93), ganglioglioma (18), ependymoma (32), medulloblastoma (22), atypical teratoid rhabdoid tumor (12), and craniopharyngioma (16). Among these samples were 19 pairs from pre/post recurrence. Across this sample set, we quantified 9155 proteins and 13632 phospho sites. After data preprocessing including normalization, batch correction and missing value imputation, we performed consensus clustering and showed that protein profiles can distinguish major histology types. However, additional regression based differential analyses revealed groups of proteins and pathways showing distinct activity patterns across different histologic types, including the novel proteomic specific clustering of V600E BRAF mutant low grade astrocytomas and non-BRAF mutant adamantinomatous craniopharyngioma.

Thus, the incorporation of the proteomic dimension into large-scale efforts at tumor characterization adds novel functional insight that links MAPK functionality in pediatric craniopharyngiomas in ways previously thought to be restricted to BRAF-mutant adult papillary craniopharyngiomas. 


\title{
Prospective Follow-Up of Anterior Vertebral Body Tethering for Idiopathic Scoliosis: Interim Results from an FDA IDE Study
}

\author{
Amer Samdani, Josh Pahys, Robert Ames, Harsh Grewal, Glenn Pelletier, \\ Randal Betz, Steven Hwang
}

\section{Introduction}

Anterior vertebral body tethering (aVBT) has emerged as a novel treatment option for patients with idiopathic scoliosis. We present results from the first FDA IDE study on aVBT.

\section{Methods}

Eligible patients underwent aVBT at a single center from August 2011 to July 2015. Inclusion criteria included skeletally immature patients with Lenke $1 \mathrm{~A}$ or $\mathrm{B}$ curves between $30^{\circ}-65^{\circ}$. Clinical and radiographic parameters were collected until completion of the study ( $>18$ years of age), with the latter measured by an independent reviewer.

\section{Results}

Fifty-five (55) patients were enrolled in the study ( $F: 47 ; M: 8)$ with mean age of $12.5 \pm 1.3$ years. Patients underwent a mean number of $7.5 \pm 1.2$ levels tethered with a mean operative time of $223 \pm 80$ minutes and EBL of $108 \pm 87 \mathrm{ml}$. Fifty-three (53) patients had minimum 2 years of followup averaging $47.8 \pm 14.3$ months with mean Risser sign at follow-up of $4.3 \pm 0.8$ (range $0-5$ ). A mean preoperative main thoracic Cobb angle of $39.7 \pm 6.8^{\circ}$, corrected to $18.9 \pm 8.1^{\circ}$ at first erect. At most recent follow-up, the Cobb angle further improved to $16.2 \pm 14.0^{\circ}$ (54\% correction). In the sagittal plane T5-12 kyphosis measured $15.1 \pm 9.6$ preop, $16.3 \pm 8.9$ postop, and $17.3 \pm 12.4$ at most recent follow-up. At most recent follow-up SRS scores averaged $4.5 \pm 0.4$, and self-image scores averaged 4.4 \pm 0.6 .

No major neurologic or pulmonary complications occurred. However, five patients (9.4\%) underwent revision surgery, four for overcorrection requiring cutting of the tether and one for adding on.

\section{Conclusion}

Anterior VBT is a promising technique that has emerged as a treatment option for patients with immature idiopathic scoliosis. These results represent the first results from a FDA approved IDE study with a mean follow-up greater than 3 years. The findings affirm the safety and efficacy of this technique, with opportunities for improvement with respect to reoperation rates. 


\title{
The Futility of Pial Closure in Spinal Lipomas
}

\author{
Michael Burke, MD
}

During the 2014 meeting of the ASPN in Costa Rica the author presented a novel classification of spinal lipomas based upon their embryologic origin from primary versus secondary neurulation. This presentation was derived from a surgical series of about 150 patients and resulted in a negligible rate of tethering. Examples of each type of lipoma were demonstrated as well as the general surgical principles in dealing with them. This included an osteoplastic laminotomy, debulking of the lipoma with no attempt for a complete and total resection of all fat cells, localization and section of the filum in lipomas derived from primary neurulation, and indicating that closure of the pia is not required and, in fact, likely contraindicated. To this date the latter recommendation has never been adopted and it remains the standard of care to close the pia. Since that time there have been several other series presented. Each of these has had a significant incidence of tethering. One of the more recent studies out of Toronto had a rate of early tethering of about $30 \%$. This led them to suggest that prophylactic surgery perhaps is not indicated and intervention should be held until the patient is demonstrating neurological symptoms. In all series reported the pia was closed.

Pial closure has historically been performed with the thought that this decreases tethering. It however, has never been shown that an extra medullary layer of fat on a spinal cord, which is floating in a CSF closed tube, will tether. That layer of fat however can potentially expand over time. With closure of the pia we now have an intramedullary spinal cord expanding mass. It is the opinion of this author that that is probably the etiology of tethering. The rate of tethering in various series is prompting some institutions to take a very aggressive approach in resecting all fat, leaving exposed spinal cord (neurological deficits) all for the benefit of continuing to close the pia. That might be fine if in fact it were possible.

The current series now involves over 400 patients. The rate of tethering remains negligible. Presented herein are examples of complex lipomas in which complete fat resection is not practical or even possible. A novel technique to maximally debulk the lipoma will be presented. As continues to be the practice no patient had the pia closed. Even in these patients, for which the traditional reported manner of closure is not possible, there has been no tethering. This leads to the logical question, that if that is the case in these complex patients, what is the logic in pial closure even in the more straightforward ones. The point to be driven home is that the dogma of hyper aggressive fat removal and pial closure continues to produce an acceptable rates of tethering. It is hoped that the information presented here will be compelling enough to alter a change in surgical approach, specifically stop closing the pia, and start a new series of cases to follow. 


\section{Chiari Malformation 0.5: Novel Entity Defines Medullary Symptoms in Young Children}

Jeffrey Greenfield, MD

\section{Objective}

Cervico-medullary compression in young children has been described in the context of Chiari type-1 malformation (CM I), with symptoms associated with the extent of tonsillar herniation below McRae's line. Historically, CM I has been defined by tonsillar herniation of at least $5 \mathrm{~mm}$. However, in certain populations including very young children, Chiari symptomatology may be present despite a lack of anatomical herniation of at least 5 raising questions about historical classification.

\section{Methods}

Patients up to five years of age evaluated for possible posterior fossa decompression were retrospectively reviewed. Pre-operative symptoms, MR-imaging, surgical management, and short-term and long-term outcome and follow-up were recorded. Tonsillar descent and presence of ventral herniation $(\mathrm{VH})$ were recorded. We define $\mathrm{VH}$ as the tonsils crossing a line that bisects the caudal medulla at the level of the foramen magnum, thus defining a novel entity, Chiari 0.5. Patients with ventrally herniated tonsils were compared with those exhibiting more typical radiographically-defined Chiari morphology.

\section{Results}

41 patients were retrospectively reviewed; 20 met criteria for Chiari 0.5. These differed from those without VH because of the predominance of medullary symptoms. In the Chiari 0.5 cohort, 11 patients underwent surgical decompression with symptom resolution; 9 were initially managed conservatively, but a third of these children required surgery at a later date.

\section{Conclusions}

We define a novel Chiari entity, Chiari 0.5 , characterized by ventral tonsillar wrapping around the medulla in young children, in the absence of classic CM I imaging findings. These patients are more likely to present with medullary symptoms and to require surgical decompression than those without $\mathrm{VH}$ and respond very favorably to surgery, suggesting adapting historical classification to include newer anatomic definitions. 


\section{Building a Clinical Research Infrastructure for Surgeons: "What's Past is Prologue"}

Jay Wellons, MD, MSPH, Stephen Gannon, CCRP, and Chevis Shannon, MPH, MBA, DrPH

Division of Pediatric Neurosurgery, Vanderbilt University School of Medicine

The Surgical Outcomes Center for Kids, Vanderbilt University School of Medicine

Surgeons are increasingly called upon to drive the clinical margin at academic medical centers across North America. Inherent in the daily clinical work is the education of the next generation of surgeons. Scholarship as noted by academic productivity has become a relevant metric in education as mandated by the most recent iteration of the ACGME's Common Program Requirements. Role conflict, however, arises as the hospital promotes higher case volumes in pursuit of revenue and therefore as a result de-emphasizes discovery and the role of clinical research. "Why would we want you to do research," one administrator noted, "when we need you in the OR and clinics."

In 2015, the Surgical Outcomes Center for Kids (SOCKs) was formed in order to promote clinical research success among pediatric surgical subspecialists at Vanderbilt. Its goal: increase academic output, level of evidence, and grant submission. SOCKs is comprised of all pediatric surgical subspecialties hospital-wide, and the overarching goal is to: 1) assess surgical and patient-centered outcomes that can inform future clinical decision making, 2) identify standards of excellence and best practices that can improve overall patient health, and 3) implement quality improvement initiatives creating opportunities for effective resource utilization for the healthcare system as a whole

The leadership of SOCKs includes a Center Director, Medical Director, and Program Director. SOCKs personnel provide support for all regulatory, oversight, recruitment and data dissemination activities to facilitate projects from start to finish. The research team consists of full-time research managers, data managers, technical writers, experienced grant writing staff, epidemiologists, nurse researchers, clinical trialists, and research fellows.

Since 2015, SOCKS has initiated over 178 clinical research studies, engaging over 30 academicallyproductive surgeon/physician scientists. $75 \%$ of our studies represent RCTs, prospective quality of life and patient-centered, interrater reliability, comparative effectiveness, cost analysis, machine learning, educational interventions, and QI initiatives. Our participation in multiinstitutional research networks has grown by $400 \%$, and has garnered approximately 4 million dollars in collaborative funding. We recently surpassed the 130 mark for peer-reviewed publication, and the proportion of abstracts submissions accepted to national and international meetings is $90 \%$. Additionally, SOCKs maintains a training arm, including a Summer Research Institute, providing clinical research training for over 85 medical and graduate students, residents 
$43^{\text {rd }}$ Annual Meeting

The American Society of Pediatric Neurosurgeons

Jointly provided by AANS
American

Association of

Neurological

Surgeons

Jointly Provided by the AANS

and clinical fellows, and representing more than 15 nationally ranked institutions across the United States. This work has resulted in 9 trainee research awards.

The presentation if accepted would report on the initial success metrics and results of the Vanderbilt Pediatric Neurosurgery Clinical Research Initiative - the precursor of SOCKs, SOCKs itself, and now the recently created VUMC Section of Surgical Sciences Clinical Research Arm for all surgeons, pediatric and adult. The presenter would discuss strategies that have led to successes and lessons learned from failure. If time allows for discussion, the audience would be actively encouraged to share their own institutional experiences with similar efforts. 


\title{
Use of iCT and Stealth AXiEM Limit Variability in a Pig Model of TBI and Stroke
}

\author{
Bauer DF, Everitt A, Calnan D, Root B, Halter R. \\ Dartmouth-Hitchcock Medical Center \\ Thayer School of Engineering
}

Animal models provide a useful bridge to preclinical studies in humans. We have developed a reproducible model of a focal hematoma and a reproducible model of MCA stroke in a Yucatan pig. The model has been facilitated by the use of intraoperative CT and electromagnetic intracranial navigation housed in a dual-use (animal/human) operating room suite at our institution. We describe the benefit of continuous intraoperative CT and EM navigation to produce and document reliable injuries between animal subjects. We propose that the construction of additional dual-use operating facilities such as this may help lead to surgical advances in the future. 


\title{
Estimating the Number of Neurosurgical Interventions in Adults with Spina Bifida using the Health Facts Dataset: Implications for Transition Planning and the Development of Adult Clinics
}

\author{
Michael D Partington MD, $\mathrm{MS}^{1}$ \\ Joseph S Domino MD, MPH ${ }^{1}$ \\ Earl F Glynn² \\ Neurosurgery ${ }^{1}$ and Research Informatics, ${ }^{2}$ Children's Mercy Hospital Kansas City and Department \\ of Neurosurgery, ${ }^{1}$ University of Kansas School of Medicine
}

\section{Purpose}

To estimate the number/frequency of neurosurgical interventions in children and adults with spina bifida, using a single large dataset.

\section{Methods}

This study used Health Facts, a de-identified proprietary dataset abstracted from all Cerner electronic health records (Cerner Corp, N Kansas City, MO). The dataset includes 69 million unique patients with over 500 million encounters in 580 centers. Diagnostic and demographic information is available from 2000-2017 and CPT4 codes from 2013-2017. The ICD-9/ICD-10 codes for all types of spinal dysraphism were searched and records retrieved. Records were sorted into groups by age (pediatric age 2-18, adult age 19-90 and a total set). Interventions were grouped by type (hydrocephalus, tethered cord and/or Chiari).

\section{Results}

24,764 unique patients were identified. The overall cohort was $57.7 \%$ female. Pediatric group included 11084 patients (51.0\% female) and adult group 14304 patients ( $62.5 \%$ female). Annual encounters were stable from age $2-18$, but then decreased by approximately half, suggesting patients either leave their original pediatric care system and enter another system (with a different EHR) or that they discontinue routine follow up. The gender distribution of adults and children undergoing procedures was similar (54.6\% female in adults vs. $52.4 \%$ female in childhood), suggesting that males return for care when necessary.

Surgical interventions in adults were common. Between 2013-2017, there were a total of 6359 procedures for hydrocephalus, with 2435 (38.3\%) adult patients. Similarly, $273(25.3 \%)$ of the 1080 tethered cord procedures were performed on adults, as were 307 (30.0\%) of 1022 Chiari decompressions.

\section{Conclusions}

This data base offers another option for studying care delivery in the transitioning spina bifida patient population. The median age of this population is now in early adulthood, and a significant number of neurosurgical procedures are performed on adults. An abrupt drop in the rate of encounters occurs at age 21 , possibly reflecting transition issues.

The authors have no financial disclosures. 


\title{
Gender disparities in Neurosurgery: A Real Pipeline?
}

\author{
Zulma Tovar Spinoza ${ }^{1}$, Nelci Zanon ${ }^{2}$ \\ ${ }^{1}$ Department of Neurosurgery, SUNY Upstate Medical University, Syracuse, NY USA \\ ${ }^{2}$ Department of Neurosurgery, Federal University of Sao Paulo, Brazil
}

\section{Objective}

To collect and analyze the data about the experiences of women in academic and non-academic neurosurgery around the world and better understand the factors that have impacted women's ability to advance in their neurosurgical careers.

\section{Material-Methods}

A survey was designed to collect data on Demographics (age, marital status, children, ethnicity, higher degree, country and continent of practice, number of female residents and attending neurosurgeon in your department, academic rank, fellowship trained, sub-specialty, years in practice, type of practice), Workload (Hours work/week, OR days/month, Clinic days/week, Academic days/week, time for completing paperwork from work at home), Research Productivity ( Life-time per-reviewed publications, factors that determine your ability to publish, applied for a grant), Career Satisfaction (Career decisions, rate of career progression, best aspects of being a neurosurgeon, worst aspects of being a neurosurgeon, what changes would attract more female to neurosurgery),Work/Personal Life Balance (Hours of sleep, partner cooperation with housekeeping, colleagues and family support in personal situations, interaction with colleagues, pregnancy), Opportunities ( professional interactions, salary gap, opportunities and promotion), Mentorship (having a mentor, being trained as a mentor). Leadership ( participation in organized neurosurgery). The survey was shared via Google Drive and was responded anonymously.

\section{Results}

Over 150 replies have been recibed so far, The Statistics will be presented and discussed.

\section{Conclusion}

The survey allows to obtain concrete responses about the different aspects of gender disparity in Neurosurgery and will help creating proposals that could be applied locally and internationally to improve the gender-related gap and pipelines.

\section{Keywords}

Gender disparity, Neurosurgery practice, glass ceiling, gap, pipeline. 


\section{Unlikely to be Abuse: Lean In, Pediatric Neurosurgeon!}

Saadi Ghatan, MD

Short falls, extra-axial fluid collections, small acute on chronic subdural hematomas, seizures, and other self-limited nervous system diagnoses seen in the pediatric emergency room can lead to forcible removal of a child from their parents. Family defense attorneys are called upon to litigate hearings in order to exculpate parents and reunite families, often with little or no background or understanding of these medical problems. Families are traumatically separated for weeks, months, and occasionally years while child protective service agencies pursue a 'better safe than sorry' approach that ignores the harm separation and even short stays in foster care bring to children.

The authors describe their experiences in the past 10 years with 27 cases of isolated nervous system diagnoses, mostly in the New York Metropolitan area. A range of injuries and presentations primarily involving seizures, benign extra-axial fluid collections, and linear skull fractures resulted in accusations of non-accidental trauma and forcible removal of children from their parents. All families were black, Latino, or of immigrant demographic backgrounds.

Direct involvement of a pediatric neurosurgeon in reviewing the medical records and imaging, and in some cases testifying at hearings and at trial, contributed to a reversal of charges and return of the children to the home. In the vast majority of cases, child protective service agencies had little to no medical evidence to support their claims of abuse. No evidence of recidivism was seen at follow up (mean 3 years, range 6 months to 10 years) in any of these cases.

The authors conclude that direct involvement of a pediatric neurosurgeon is critical in helping to assess the unlikelihood of non-accidental trauma. In the setting of self-limited neurological diagnoses such as linear skull fractures and benign extra-axial fluid collections, a pro-active collaboration between pediatric neurosurgeons, child abuse pediatricians, pediatric emergency room staff, and family defense attorneys can help restore order in the court. 


\title{
Uncertainty, Fear, Communication and Loss of Relationships: A Qualitative Study of Patients and Families with Hydrocephalus Transitioning from Pediatric to Adult Care
}

\author{
Saman Fouladirad BSc ${ }^{1}$, Alexander Cheong BSc${ }^{1}$, Mandeep Tamber MD, PhD, FRCSC ${ }^{1,2}$, Ashutosh \\ Singhal MD, MSc, FRCSC ${ }^{1,2}$, Patrick J. McDonald MD, MHSc, FRCSC ${ }^{1,2,3, *}$ \\ ${ }^{1}$ Division of Neurosurgery, BC Children's Hospital, Vancouver, BC, Canada; ${ }^{2}$ Division of Neurosurgery, Department \\ of Surgery, University of British Columbia, Vancouver, BC, Canada; ${ }^{3}$ Neuroethics Canada, University of British \\ Columbia, Vancouver, BC, Canada; *Presenting and corresponding author
}

\section{Background}

Hydrocephalus is the most common condition treated by pediatric neurosurgeons but many are unable to continue to care for their patients after they become adults. Although significant gaps in care are thought to exist for youth transitioning from pediatric to adult care, very little is known about how patients and their caregivers feel about the process. This study sought to examine the perceptions of adolescents and young adults transitioning from pediatric to adult care at a single Canadian center.

\section{Methods}

We explored the perceptions of patients with treated hydrocephalus and their caregivers using semi-structured interviews and the qualitative research methodologies of grounded theory. We recruited a convenience sample of adolescent patients and their caregivers at the neurosurgery clinic at BC Children's Hospital or patients and caregivers recently transitioned to adult care from the clinic. Interviews were transcribed verbatim and coded, with common themes identified.

\section{Results}

Four overarching themes relating to the process of transitioning from pediatric to adult hydrocephalus care for patients and their caregivers were identified from the data: (I): Inadequacy of communication between pediatric and adult neurosurgeons and patients and families: (2) Uncertainty relating to the prospect of living life as an adult with hydrocephalus, especially among those with developmental challenges; (3) Anxiety and fear regarding navigating a new health care environment; and (4) sadness in the loss of the relationship with the pediatric health care team.

\section{Conclusions}

Overall, patients with hydrocephalus and their families are dissatisfied with the process of transitioning. We identified common themes and concerns among this cohort that may form the basis of an improved transitioning model for youth with hydrocephalus as they become adults.

\section{Disclosures}

None of the authors have any disclosures relevant to this study'ynauthenticated | Downloaded 04/26/23 10:35 AM UTC 


\title{
The ACS Children's Surgery Verification (Quality Improvement Program) - Origins and Discussion of its Current Impact/Interaction with Pediatric Neurosurgery Care
}

\author{
Bruce A Kaufman, MD \\ Children's Hospital of Wisconsin - Medical College of Wisconsin \\ Milwaukee, WI \\ (contact: bkaufman@mcw.edu)
}

In early 2012 a leading pediatric surgeon identified that individual patient needs and available clinical resources for some infants and children receiving surgical care were mismatched. He assembled an ad hoc group of pediatric surgical and associated specialists to review the published literature and data, and when necessary to discuss missing information using an 'expert opinion' approach. Over four years, that group created and published a consensus document to be "of use to relevant policy makers and providers".

An immediate result of this work (if not the true goal of the consensus effort) was the development of a Children's Surgery Verification and Quality Improvement Program under the direction of the American College of Surgeons. This allowed facilities to apply for and receive verified status as a Level I, II or III pediatric surgical center - after meeting criteria specific for each level. Recently, some institutions have applied for Level I status and in the process concerns have been raised around the ACS verification criteria and how they apply to pediatric neurosurgical care.

This presentation will review the origins of this ACS program and begin a discussion of the issues affecting appropriate pediatric neurosurgical care delivery. It is based on the author's participation in the consensus statement development, and more recent observations of and discussions with the ACS leadership.

Disclosures: None 